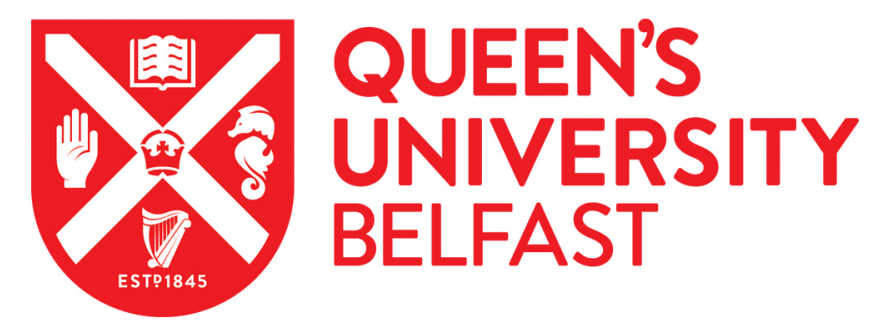

\title{
Multielectron effects in high harmonic generation in N2 and benzene: Simulation using a non-adiabatic quantum molecular dynamics approach for laser-molecule interactions
}

Dundas, D. (2012). Multielectron effects in high harmonic generation in N2 and benzene: Simulation using a non-adiabatic quantum molecular dynamics approach for laser-molecule interactions. Journal of Chemical Physics, 136(19), [194303]. https://doi.org/10.1063/1.4718590

Published in:

Journal of Chemical Physics

Document Version:

Publisher's PDF, also known as Version of record

Queen's University Belfast - Research Portal:

Link to publication record in Queen's University Belfast Research Portal

Publisher rights

(c) 2012 American Institute of Physics

\section{General rights}

Copyright for the publications made accessible via the Queen's University Belfast Research Portal is retained by the author(s) and / or other copyright owners and it is a condition of accessing these publications that users recognise and abide by the legal requirements associated with these rights.

Take down policy

The Research Portal is Queen's institutional repository that provides access to Queen's research output. Every effort has been made to ensure that content in the Research Portal does not infringe any person's rights, or applicable UK laws. If you discover content in the Research Portal that you believe breaches copyright or violates any law, please contact openaccess@qub.ac.uk. 


\section{AIP $\begin{gathered}\text { mosoumator } \\ \text { chemical Physics }\end{gathered}$}

Multielectron effects in high harmonic generation in N2 and benzene:

Simulation using a non-adiabatic quantum molecular dynamics approach for laser-molecule interactions

Daniel Dundas

Citation: J. Chem. Phys. 136, 194303 (2012); doi: 10.1063/1.4718590

View online: http://dx.doi.org/10.1063/1.4718590

View Table of Contents: http://jcp.aip.org/resource/1/JCPSA6/v136/i19

Published by the American Institute of Physics.

Additional information on J. Chem. Phys.

Journal Homepage: http://jcp.aip.org/

Journal Information: http://jcp.aip.org/about/about_the_journal

Top downloads: http://jcp.aip.org/features/most_downloaded

Information for Authors: http://jcp.aip.org/authors

\section{ADVERTISEMENT}

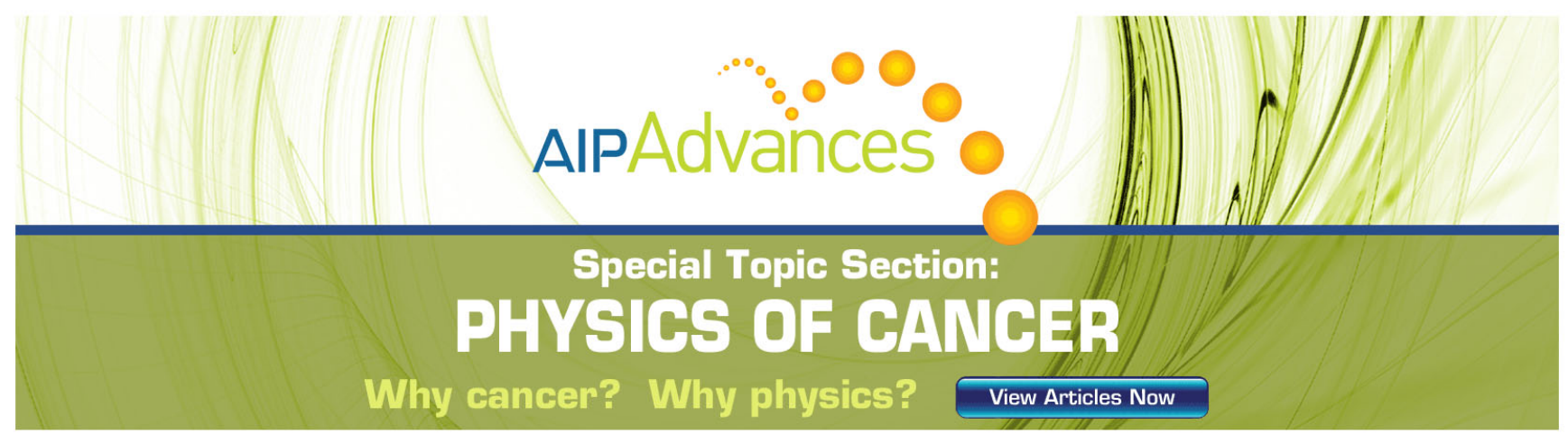




\title{
Multielectron effects in high harmonic generation in $\mathbf{N}_{\mathbf{2}}$ and benzene: Simulation using a non-adiabatic quantum molecular dynamics approach for laser-molecule interactions
}

\author{
Daniel Dundas \\ Atomistic Simulation Centre, School of Mathematics and Physics, Queen's University Belfast, \\ Belfast BT7 1NN, Northern Ireland, United Kingdom
}

(Received 21 November 2011; accepted 1 May 2012; published online 17 May 2012)

\begin{abstract}
A mixed quantum-classical approach is introduced which allows the dynamical response of molecules driven far from equilibrium to be modeled. This method is applied to the interaction of molecules with intense, short-duration laser pulses. The electronic response of the molecule is described using time-dependent density functional theory (TDDFT) and the resulting Kohn-Sham equations are solved numerically using finite difference techniques in conjunction with local and global adaptations of an underlying grid in curvilinear coordinates. Using this approach, simulations can be carried out for a wide range of molecules and both all-electron and pseudopotential calculations are possible. The approach is applied to the study of high harmonic generation in $\mathrm{N}_{2}$ and benzene using linearly polarized laser pulses and, to the best of our knowledge, the results for benzene represent the first TDDFT calculations of high harmonic generation in benzene using linearly polarized laser pulses. For $\mathrm{N}_{2}$ an enhancement of the cut-off harmonics is observed whenever the laser polarization is aligned perpendicular to the molecular axis. This enhancement is attributed to the symmetry properties of the Kohn-Sham orbital that responds predominantly to the pulse. In benzene we predict that a suppression in the cut-off harmonics occurs whenever the laser polarization is aligned parallel to the molecular plane. We attribute this suppression to the symmetryinduced response of the highest-occupied molecular orbital. () 2012 American Institute of Physics. [http://dx.doi.org/10.1063/1.4718590]
\end{abstract}

\section{INTRODUCTION}

Molecules driven out of equilibrium by external forces are of fundamental importance in many areas of science and technology. In such a non-equilibrium situation, a nonadiabatic coupling exists between electrons and ions which can induce charge and energy transfer across the molecule on a femtosecond time scale. ${ }^{1}$ These charge and energy transfer processes are of extreme importance in the design of electronic devices, ${ }^{2}$ probes and sensors, ${ }^{3}$ and in the areas of condensed matter and plasma physics, medicine and biochemistry.

Of particular interest and importance is the interaction of molecules with ultra-short laser pulses. In this case, the laser couples to the electrons in the molecule and this coupling can lead to ionization and subsequent dissociation of the molecule if the laser intensity is high enough. Indeed, even for low laser intensities current flow in molecular electronic devices can be both induced and suppressed. ${ }^{4-6}$ Over the last decade much effort has focused on the possibility of using ultra-short laser pulses to control chemical reactions. Experimental studies have already used genetic algorithms to create optimal pulse profiles in order to break specific bonds in complex molecules. ${ }^{7-9}$ In these experiments Ti:sapphire laser pulses (wavelength, $\lambda \sim 800 \mathrm{~nm}$ ) were employed with the interaction taking place over a time scale of $10 \mathrm{~s}$ of femtoseconds. Alternatively, few-cycle optical pulses have been used to steer dissociation by varying the carrier-envelope phase of the pulse. ${ }^{10-12}$ More recently, the use of attosecond pulses has been considered for controlling the electron dynamics directly. These pulses generally operate at high laser frequencies (VUV to x-ray wavelengths) and can be created either through high harmonic generation (HHG) in gases ${ }^{13-16}$ or in free-electron laser sources. Much of the work on attosecond pulses has focused on controlling processes such as electron localization ${ }^{17-19}$ as this control is seen as the crucial first step in controlling chemical reactions: a fuller review of this subject is given by Krausz and Ivanov. ${ }^{20}$

The study of multielectron effects in the response of molecules to intense laser pulses has attracted much attention in recent years. This interest stems from the complexity of the electronic structure in molecules together with the experimental ability to control the orientation between the molecules and the laser pulse using a variety of alignment techniques. While initial studies considered multielectron effects in ionization ${ }^{21-29}$ recent studies have considered the role of multielectron effects in HHG. ${ }^{30-48}$ Studying HHG as a function of alignment provides us not only with an insight to the role of molecular electronic structure in HHG, but also with a technique for manipulating the strength of the high harmonics emitted. Indeed, the sub-femtosecond timing information encoded in these high harmonics can be used as a tool to image dynamically the molecular orbitals themselves ${ }^{49,50}$ and, if several orbitals contribute to the HHG process, sub-femtosecond electron dynamics in the molecule can be 
directly imaged ${ }^{41}$ Therefore, an understanding of multielectron effects in molecular HHG is crucial to the development of these imaging techniques.

Any model for describing the interaction of molecules with ultra-short laser pulses must be able to handle length, time and energy scales that can span several orders of magnitude. In the first instance we need to model processes occurring on time scales ranging from the sub-femtosecond for electronic motion to $100 \mathrm{~s}$ of femtoseconds for the dissociation of the molecule. Second, the laser wavelength can vary from optical to VUV wavelengths which alters how the laser couples to the electrons in the molecule: while optical pulses couple predominantly with the valence electrons in the molecule, attosecond pulses can couple directly to the innermost electrons. Finally, in describing processes such as ionization and HHG we must be able to describe accurately the emission of high-energy electrons over length scales of several hundred Bohr radii. For simple molecular systems, such as one- and two-electron diatomic molecules, solution of the time-dependent Schrödinger equation (TDSE) is possible. ${ }^{17,51-57}$ However, describing more complex molecules requires further approximation. These approximations range from simple models which handle only crucial aspects of the dynamics to full ab initio simulations of the electrons and ions in the presence of the laser pulse.

One $a b$ initio method that is widely used to treat electronic dynamics is time-dependent density functional theory (TDDFT). ${ }^{58}$ The TDDFT method has been applied extensively to the study of molecules and clusters subject to external forces. ${ }^{59-65}$ In many cases this application involves coupling the quantum treatment of the electronic degrees of freedom to a classical treatment of the nuclear degrees of freedom, resulting in a method known as the non-adiabatic quantum molecular dynamics (NAQMD) method. ${ }^{59-61}$ Implementations of NAQMD have generally used basis-set ${ }^{59-62}$ or grid $^{63-65}$ techniques. While grid approaches offer better opportunities for code parallelization, allowing efficient scaling to large problem sizes, they do suffer from the drawback of requiring small grid spacings in order to deal with moving ions on the grid. This problem can be overcome by using grid adaptation techniques employing both local and global adaptations. ${ }^{52,66-75}$

In this paper we set out our NAQMD approach on a real space grid that allows the use of both local and global adaptations. The novelty of the approach lies in the range of methods that are implemented and that, in implementing these methods together, a wide range of problems can be studied. The approach can handle either all of the electrons or only a subset, depending on the laser-pulse used, and its implementation opens up the possibility of carrying out ab initio simulations of the response of complex molecules to intense laser pulses of arbitrary polarization. This is in contrast to many other implementations of TDDFT which have been optimised to efficiently study ionization and HHG in either diatoms irradiated by linearly polarized light ${ }^{39,46}$ or in polyatomic molecules, such as benzene, irradiated by circularly polarized light. ${ }^{32}$ The paper is arranged as follows. In Sec. II a set of equations of motion for a system of quantum mechanical electrons and classical ions is derived using a Lagrangian formalism. This derivation allows for velocity-dependent terms to be introduced in cases where either a moving basis or moving grid is introduced. In Sec. III the equations of motion are re-expressed whenever TDDFT is used to describe the electronic system. A number of local and global coordinate transformations are described in Sec. IV which will allow a standard finite difference grid to be warped in different regions of space. Section V describes how the NAQMD approach is implemented in a real-space code. In Sec. VI the method is applied to study the influence of multielectron effects in HHG in molecules. In particular, our calculations for HHG in benzene predict a suppression of the high-order harmonics as the alignment between the molecular plane and the laser polarization direction varies. Some conclusions are drawn in Sec. VII.

Unless otherwise stated, atomic units are used throughout.

\section{THE NON-ADIABATIC QUANTUM MOLECULAR DYNAMICS APPROACH}

We consider a system consisting of $N_{e}$ quantummechanical electrons and $N_{n}$ classical ions. The electrons are described by their many-body wavefunction $\Psi\left(\boldsymbol{r}_{e}, t\right)$, where $\boldsymbol{r}_{e}=\left\{\boldsymbol{r}_{1}, \ldots, \boldsymbol{r}_{N_{e}}\right\}$ denotes the electron position vectors (for the time being we will ignore spin). The ions are described by their trajectories $\boldsymbol{R}=\left\{\boldsymbol{R}_{1}(t), \ldots, \boldsymbol{R}_{N_{n}}(t)\right\}$ and momenta $\boldsymbol{P}=\left\{\boldsymbol{P}_{1}(t), \ldots, \boldsymbol{P}_{N_{n}}(t)\right\}$. For ion $k$, we denote its mass and charge by $M_{k}$ and $Z_{k}$, respectively.

In order to derive a set of equations of motion for the electrons and ions we will use a Lagrangian formalism. ${ }^{76,77}$ We start from the Lagrangian

$$
\begin{aligned}
\mathcal{L}= & \mathrm{i} \int d \boldsymbol{r}_{e} \Psi^{\star}\left(\boldsymbol{r}_{e}, t\right) \dot{\Psi}\left(\boldsymbol{r}_{e}, t\right) \\
& -\int d \boldsymbol{r}_{e} \Psi^{\star}\left(\boldsymbol{r}_{e}, t\right) H\left(\boldsymbol{r}_{e}, \boldsymbol{R}, t\right) \Psi\left(\boldsymbol{r}_{e}, t\right) \\
& +\frac{1}{2} \sum_{k=1}^{N_{n}} M_{k} \dot{\boldsymbol{R}}_{k}^{2}(t)-V_{n n}(\boldsymbol{R}),
\end{aligned}
$$

where

$$
V_{n n}(\boldsymbol{R})=\sum_{k<k^{\prime}}^{N_{n}} \frac{Z_{k} Z_{k^{\prime}}}{\left|\boldsymbol{R}_{k}-\boldsymbol{R}_{k^{\prime}}\right|},
$$

denotes the Coulomb repulsion between the ions and $H\left(\boldsymbol{r}_{e}, \boldsymbol{R}, t\right)$ is the time-dependent Hamiltonian for the electrons which depends parametrically on the ion coordinates. The Hamiltonian can be written as

$$
H\left(\boldsymbol{r}_{e}, \boldsymbol{R}, t\right)=\sum_{i=1}^{N_{e}}\left[-\frac{1}{2} \nabla_{i}^{2}+V_{\mathrm{ext}}\left(\boldsymbol{r}_{i}, \boldsymbol{R}, t\right)\right]+V_{e e}\left(\boldsymbol{r}_{e}\right),
$$

where $\nabla_{i}^{2}$ is the Laplacian with respect to the coordinates of electron $i$. In this Hamiltonian,

$$
V_{e e}\left(\boldsymbol{r}_{e}\right)=\sum_{i<j}^{N_{e}} \frac{1}{\left|\boldsymbol{r}_{i}-\boldsymbol{r}_{j}\right|}
$$


is the Coulomb repulsion between electrons and

$$
V_{\mathrm{ext}}\left(\boldsymbol{r}_{i}, \boldsymbol{R}, t\right)=V_{\mathrm{ions}}\left(\boldsymbol{r}_{i}, \boldsymbol{R}, t\right)+U_{\mathrm{elec}}\left(\boldsymbol{r}_{i}, t\right)
$$

is the external potential consisting of $U_{\text {elec }}\left(\boldsymbol{r}_{i}, t\right)$, the interaction between electron $i$ and the applied laser field, and

$$
\begin{aligned}
V_{\text {ions }}\left(\boldsymbol{r}_{i}, \boldsymbol{R}\right) & =\sum_{k=1}^{N_{n}} V_{\mathrm{ion}}\left(\boldsymbol{r}_{i}, \boldsymbol{R}_{k}\right) \\
& =-\sum_{k=1}^{N_{n}} \frac{Z_{k}}{\left|\boldsymbol{r}_{i}-\boldsymbol{R}_{k}\right|},
\end{aligned}
$$

the Coulomb interaction between electron $i$ and all ions. Additionally, in Eq. (1)

$$
\int d \boldsymbol{r}_{e}=\int d \boldsymbol{r}_{1} \cdots \int d \boldsymbol{r}_{N_{e}}
$$

refers to integration over all electron coordinates.

The equations of motion for the electrons and ions can be obtained by considering variations of the wavefunction and ion trajectories that leave the action, $\mathcal{A}$, stationary, i.e.,

$$
\delta \mathcal{A}=\delta \int_{t_{0}}^{t_{1}} \mathcal{L} d t=0 .
$$

This results in the Euler-Lagrange equations of motion

$$
\begin{gathered}
\frac{\partial \mathcal{L}}{\partial \Psi^{\star}}=\frac{d}{d t}\left(\frac{\partial \mathcal{L}}{\partial \dot{\Psi^{\star}}}\right), \\
\frac{\partial \mathcal{L}}{\partial \Psi}=\frac{d}{d t}\left(\frac{\partial \mathcal{L}}{\partial \dot{\Psi}}\right), \\
\frac{\partial \mathcal{L}}{\partial \boldsymbol{R}_{k}}=\frac{d}{d t}\left(\frac{\partial \mathcal{L}}{\partial \dot{\boldsymbol{R}}_{k}}\right) .
\end{gathered}
$$

Equation (9) leads to the TDSE

$$
\mathrm{i} \frac{\partial}{\partial t} \Psi\left(\boldsymbol{r}_{e}, t\right)=H\left(\boldsymbol{r}_{e}, \boldsymbol{R}, t\right) \Psi\left(\boldsymbol{r}_{e}, t\right),
$$

while Eq. (10) gives its complex conjugate. Equation (11) leads to the equation of motion for the ions

$$
\begin{aligned}
M_{k} \ddot{\boldsymbol{R}}_{k}= & -\int d \boldsymbol{r}_{e} \Psi^{\star}\left(\boldsymbol{r}_{e}, t\right)\left(\tilde{\nabla}_{k} H\left(\boldsymbol{r}_{e}, \boldsymbol{R}, t\right)\right) \Psi\left(\boldsymbol{r}_{e}, t\right) \\
& -\tilde{\nabla}_{k} V_{n n}(\boldsymbol{R}),
\end{aligned}
$$

where $\tilde{\nabla}_{k}$ denotes the gradient operator with respect to the ionic coordinates of ion $k$.

The Lagrangian formalism has the benefit of allowing the equations of motion for electrons and ions to be easily derived in situations where either a finite basis set is used or where dynamical locally-adaptive grids - in which the grid is adapted in a small region around the instantaneous ionic positions are used. For instance, when an incomplete basis set of atomcentred Gaussian functions is used, the resulting equations of motion have been derived by several authors. ${ }^{59,76,77}$ In that case, velocity-dependent forces (the so-called Pulay forces ${ }^{78}$ ) are introduced. The use of dynamical, locally adaptive grids will also introduce Pulay-type forces on the ions. This point will be returned to in Sec. IV.
It is important to note that a mixed quantum-classical description of electron-ion dynamics has a number of drawbacks. For example, a classical description of heavy ions can be justified when considering processes which occur over a time scale of several femtoseconds. However, when considering processes that involve light ions (such as hydrogen atoms) quantum effects can become important, in which case a classical description will no longer be appropriate. This is particularly true when considering the response of simple diatomic molecules, such as $\mathrm{H}_{2}^{+}$and $\mathrm{H}_{2}$, to intense laser pulses. For these systems, quantum treatments of electrons and ions can be carried out and these clearly show the importance of quantum nuclear motion. ${ }^{54,79-82}$ For instance, Bandrauk and coworkers clearly show that a a quantum description of nuclear motion is important for $\mathrm{HHG}$ in $\mathrm{H}_{2}^{+} .82$

\section{TIME-DEPENDENT DENSITY FUNCTIONAL TREATMENT OF THE ELECTRON DYNAMICS}

In Sec. II we derived a set of equations of motion for a system of quantum mechanical ions and classical ions. The TDSE derived for the many-body electronic wavefunction can only be solved in full dimensionality for the simplest few body systems. In this section we will consider a TDDFT description of electronic structure. ${ }^{58}$

In an earlier paper a set of equations of motion was derived for the electrons and ions using a static real-space grid. ${ }^{63}$ Here, we derive the equations of motion, using the Lagrangian formalism, for the Kohn-Sham orbitals in TDDFT that will allow us to consider the more general case of a grid that can deforms around the instantaneous ion positions. In the TDDFT method, the total $N_{e}$-electron Kohn-Sham wavefunction is written as a single determinant of electron orbitals $\psi_{i \sigma}(\boldsymbol{r}, t)$ with the electron density given by

$$
n(\boldsymbol{r}, t)=\sum_{\sigma=\downarrow, \uparrow} n_{\sigma}(\boldsymbol{r}, t)=\sum_{\sigma=\downarrow, \uparrow} \sum_{i=1}^{N_{\sigma}}\left|\psi_{i \sigma}(\boldsymbol{r}, t)\right|^{2},
$$

where $N_{\sigma}$ is the number of Kohn-Sham orbitals for spin state $\sigma$ and $N_{e}=N_{\downarrow}+N_{\uparrow}$.

In order to derive the equations of motion we consider the Lagrangian

$$
\begin{aligned}
\mathcal{L}= & \mathrm{i} \sum_{\sigma=\downarrow, \uparrow} \sum_{i=1}^{N_{\sigma}} \int d \boldsymbol{r} \psi_{i \sigma}^{\star}(\boldsymbol{r}, t) \dot{\psi}_{i \sigma}(\boldsymbol{r}, t) \\
& +\frac{1}{2} \sum_{\sigma=\downarrow, \uparrow} \sum_{i=1}^{N_{\sigma}} \int d \boldsymbol{r} \psi_{i \sigma}^{\star}(\boldsymbol{r}, t) \nabla^{2} \psi_{i \sigma}(\boldsymbol{r}, t) \\
& -\int d \boldsymbol{r} n(\boldsymbol{r}, t)\left(V_{\mathrm{ext}}(\boldsymbol{r}, \boldsymbol{R}, t)+\frac{1}{2} \int d \boldsymbol{r}^{\prime} \frac{n\left(\boldsymbol{r}^{\prime}, t\right)}{\left|\boldsymbol{r}-\boldsymbol{r}^{\prime}\right|}\right) \\
& -A_{\mathrm{xc}}\left[n_{\downarrow}, n_{\uparrow}\right] \\
& +\frac{1}{2} \sum_{k=1}^{N_{n}} M_{k} \dot{\boldsymbol{R}}_{k}^{2}(t)-V_{n n}(\boldsymbol{R}) .
\end{aligned}
$$

In this equation, $A_{\mathrm{xc}}\left[n_{\downarrow}, n_{\uparrow}\right]$ is the exchange-correlation action functional of TDDFT. The equations of motion for the KohnSham orbitals and ions can be obtained in a similar fashion 
to Eqs. (9)-(11) with the many-body wavefunction, $\Psi^{\star}$, in Eq. (9) replaced by the Kohn-Sham orbitals, $\psi_{i \sigma}^{\star}(\boldsymbol{r}, t)$. From these Euler-Lagrange equations we obtain the time-dependent Kohn-Sham equations

$$
\mathrm{i} \frac{\partial}{\partial t} \psi_{i \sigma}(\boldsymbol{r}, t)=H_{\mathrm{ks}, \sigma}(\boldsymbol{r}, \boldsymbol{R}, t) \psi_{i \sigma}(\boldsymbol{r}, t) .
$$

In this equation,

$$
H_{\mathrm{ks}, \sigma}(\boldsymbol{r}, \boldsymbol{R}, t)=-\frac{1}{2} \nabla^{2}+V_{\mathrm{eff}, \sigma}(\boldsymbol{r}, \boldsymbol{R}, t)
$$

is the Kohn-Sham Hamiltonian and

$$
V_{\mathrm{eff}, \sigma}(\boldsymbol{r}, \boldsymbol{R}, t)=V_{\mathrm{ext}}(\boldsymbol{r}, \boldsymbol{R}, t)+V_{\mathrm{H}}(\boldsymbol{r}, t)+V_{\mathrm{xc}, \sigma}(\boldsymbol{r}, t),
$$

where

$$
V_{\mathrm{H}}(\boldsymbol{r}, t)=\int d \boldsymbol{r}^{\prime} \frac{n\left(\boldsymbol{r}^{\prime}, t\right)}{\left|\boldsymbol{r}-\boldsymbol{r}^{\prime}\right|},
$$

is the Hartree potential, $V_{\text {ext }}(\boldsymbol{r}, \boldsymbol{R}, t)$ is defined from Eq. (5) and $V_{\mathrm{xc} \sigma}(\boldsymbol{r}, t)$ is the exchange-correlation potential.

The corresponding equations of motion for the ions are obtained from Eq. (11) and are analogous to those obtained in Eq. (13), namely,

$$
\begin{aligned}
M_{k} \ddot{\boldsymbol{R}}_{k}= & -\sum_{\sigma=\downarrow, \uparrow} \int d \boldsymbol{r} n_{\sigma}(\boldsymbol{r}, t)\left(\tilde{\nabla}_{k} H_{\mathrm{ks}, \sigma}(\boldsymbol{r}, \boldsymbol{R}, t)\right) \\
& -\tilde{\nabla}_{k} V_{n n}(\boldsymbol{R}) .
\end{aligned}
$$

At this point we have a set of equations of motion for electrons and ions which introduce a non-adiabatic coupling between the two subsystems. We now show how these equations can be solved numerically using a real-space grid in adaptive curvilinear coordinates.

\section{ADAPTIVE CURVILINEAR COORDINATES}

Adaptive curvilinear coordinates have been widely used in electronic structure calculations, both in plane-wave basisset methods ${ }^{66-70}$ and real-space grid techniques. ${ }^{52,71-75,83}$ In this approach either a local or global warping of space is carried out and in some situations a combination of both is used. In finite difference methods this warping of space leads to a high density of grid points around the centres of adaptation while in plane-wave methods the warping gives rise to an effective energy cut-off which varies locally. ${ }^{84}$

In general, we will consider the physical space to be described by Cartesian coordinates and then consider a transformation to a set of generalised curvilinear coordinates (which may, or may not, be orthogonal) that act as the computational space. The Kohn-Sham equations will then be solved in these curvilinear coordinates using finite difference techniques (see Sec. V A). In this scenario, the grid points in curvilinear coordinates are equally spaced which results in efficient nearest neighbour communication patterns when the resulting computer code is parallelized (see Sec. V B).

In the following, we describe a range of global and local coordinate transformations that we have implemented. Our underlying curvilinear system will be described by the coordinates $\left(\zeta^{1}, \zeta^{2}, \zeta^{3}\right)$. Global adaptation will transform these to a set of coordinates $\left(u\left(\zeta^{1}\right), v\left(\zeta^{2}\right), w\left(\zeta^{3}\right)\right)$. A local adaptation can then be applied to these coordinates to transform to Cartesian coordinates $(x, y, z)$ so that

$$
\begin{aligned}
& \zeta^{1} \quad u\left(\zeta^{1}\right) \quad x(u, v, w)=x\left(\zeta^{1}, \zeta^{2}, \zeta^{3}\right), \\
& \zeta^{2} \Rightarrow v\left(\zeta^{2}\right) \Rightarrow y(u, v, w)=y\left(\zeta^{1}, \zeta^{2}, \zeta^{3}\right), \\
& \zeta^{3} \quad w\left(\zeta^{3}\right) \quad z(u, v, w)=z\left(\zeta^{1}, \zeta^{2}, \zeta^{3}\right) .
\end{aligned}
$$

The transformation between Cartesian and curvilinear coordinates is described by the Jacobian matrix

$$
\boldsymbol{J}=\left(\begin{array}{lll}
\frac{\partial x^{1}}{\partial \zeta^{1}} & \frac{\partial x^{1}}{\partial \zeta^{2}} & \frac{\partial x^{1}}{\partial \zeta^{3}} \\
\frac{\partial x^{2}}{\partial \zeta^{1}} & \frac{\partial x^{2}}{\partial \zeta^{2}} & \frac{\partial x^{2}}{\partial \zeta^{3}} \\
\frac{\partial x^{3}}{\partial \zeta^{1}} & \frac{\partial x^{3}}{\partial \zeta^{2}} & \frac{\partial x^{3}}{\partial \zeta^{3}}
\end{array}\right)
$$

where each element can be written as $J_{\alpha}^{i}$ and its determinant, $|J|=\operatorname{det} \boldsymbol{J}$, describes how the volume element changes. The metric in Cartesian coordinates, $g_{i j}=\delta_{i j}$, can be written in matrix form as the identity matrix, i.e., $\boldsymbol{g}=\boldsymbol{I}$. In curvilinear coordinates the metric tensor transforms to $g_{\alpha \beta}$ whose elements can be written in matrix form as $\boldsymbol{g}=\boldsymbol{J}^{T} \boldsymbol{J}$.

\section{A. Globally adaptive curvilinear coordinates}

Globally adaptive coordinates allow one particular curvilinear coordinate to be scaled independently of the others. Such scaling techniques have been widely used whenever a particular density of points is required along a given axis. For example, in the treatment of linear molecules a global scaling has been used in cylindrical coordinates to give a high density of points near the molecular axis. ${ }^{52,75,83}$ There are a number of ways to implement a global scaling. We will detail three such techniques which transform the curvilinear coordinate $\zeta$ to the scaled coordinate $u$.

1. Using the transformation

$$
u(\zeta)=\sinh \left(\frac{\zeta}{\alpha}\right)
$$

where $\alpha$ is a parameter used to control the maximum extent of the grid in $u$, we obtain a high density of grid points near the origin and a low density of grid points far from the origin.

2. Under the transformation

$$
u(\zeta)=\zeta\left(\frac{\zeta^{n}}{\zeta^{n}+\alpha^{n}}\right)^{\nu}
$$

where $n$ takes on integer values, $v$ takes on half-integer values in general and $\alpha$ is a real number, we obtain a high density of grid points near origin and an equidistant grid spacing far from the origin. ${ }^{83}$ The parameter $\alpha$ controls where the transition between the regularly spaced and densely spaced grid occurs. 
3. Consider the transformation

$$
u(\zeta)=\left\{\begin{array}{cc}
\zeta & |\zeta| \leq \zeta_{f} \\
\zeta+d_{\max }\left(\frac{\zeta-\zeta_{f}}{\zeta_{f}-\zeta_{\max }}\right)^{5} & |\zeta|>\zeta_{f}
\end{array}\right.
$$

where $d_{\max }=\zeta_{\max }-u_{\max }, \zeta_{\max }$ is the maximum value of the unscaled coordinate, $\zeta_{f}$ is the point where the flat region ends, $u_{\max }$ is the maximum value of the scaled coordinate required and where $u(-\zeta)=-u(\zeta)$ when $\zeta$ $<-\zeta_{f}$. This scaling gives rise to a grid that has an equidistant spacing near origin and a low density of grid points far from the origin. This is a specific case of the general global backdrop described by Modine et al. ${ }^{73}$ in which $u$ and its first four derivatives match at $|\zeta|=\zeta_{f}$.

When both local and global adaptations are used only the third global transformation is employed.

\section{B. Locally adaptive curvilinear coordinates}

In finite-difference electronic structure calculations the error is greatest in regions around the ions. This error is usually minimized by using a combination of high-order finite difference formulae and small grid spacings. ${ }^{85,86}$ An alternative approach is to carry out a local adaptation of the curvilinear coordinates around the ions to give a high density of points in these regions. In the following, we implement the adaptive coordinate technique used in the ACRES DFT approach of Modine et $_{\text {al. }}{ }^{73}$ and describe the transformation from generalised curvilinear coordinates $\zeta=\left(\zeta^{1}, \zeta^{2}, \zeta^{3}\right)$ to Cartesian coordinates $\boldsymbol{r}=\left(x^{1}, x^{2}, x^{3}\right)=(x, y, z)$. This procedure can be extended to incorporate the global adaptations introduced in Sec. IV A. The local transformation is

$$
\boldsymbol{r}=\boldsymbol{\zeta}-\sum_{k=1}^{N_{n}} \boldsymbol{Q}_{k} \cdot\left(\boldsymbol{\zeta}-\boldsymbol{\mathcal { R }}_{k}(t)\right) \exp \left[-\left(\frac{\boldsymbol{\zeta}-\mathcal{R}_{k}(t)}{\sigma_{k}}\right)^{2}\right]
$$

In this expression $\boldsymbol{Q}_{k}$ and $\sigma_{k}$ are parameters controlling the strength and extent of adaptation in the vicinity of ion $k$. In general each $\boldsymbol{Q}_{k}$ is a $3 \times 3$ matrix, although it is sufficient to consider each $\boldsymbol{Q}_{k}$ to be diagonal. We adjust the elements of $\boldsymbol{Q}_{k}$ to satisfy $J_{\alpha}^{i}\left(\boldsymbol{R}_{k}\right)=\left|J\left(\boldsymbol{R}_{k}\right)\right|^{1 / 3} \delta_{\alpha}^{i}$. If the adaptation regions associated with different ions overlap, each adaptation centre attempts to draw grid points to itself. As a result of this competition, the highest density of points will not be around the exact ionic positions. Therefore, we perform the adaptation around fictitious positions $\boldsymbol{\mathcal { R }}_{k}(t)$ which are chosen so that on the fully adapted grid $\boldsymbol{r}\left(\boldsymbol{\mathcal { R }}_{k}\right)=\boldsymbol{R}_{k}$. Optimal values for the elements of $\boldsymbol{Q}_{k}$ and $\boldsymbol{\mathcal { R }}_{k}$ are found self-consistently using Jacobi iteration.

The effect of this transformation is illustrated in Fig. 1 where we consider a local adaptation of a finite difference grid around the atoms in benzene, denoted by the grey circles. In Fig. 1(a) we present the Cartesian grid with no adaptation using a grid spacing of 0.4 a.u. For clarity we only present the two dimensions $x$ and $y$. Under the transformation of Eq. (26) the adapted grid is shown in Fig. 1(b). The effect of this transformation is an increase in grid density in the vicinity of the atoms. In this example we use identical adaptations around each atom. However, the form of Eq. (26) is such that each atom can have different strengths and extents of adaptation.

Obviously under this coordinate transformation, the distribution of points will change as the ions move. Since the electronic position vector will depend implicitly on the ion positions, this results in the introduction of Pulay corrections to the forces defined in Eq. (20). ${ }^{73,78}$ In addition, as the ions move the grid deformation will alter and so a grid regeneration will be required together with an interpolation of the Kohn-Sham orbitals onto this new grid. In situations where the ions do not move significantly, we can choose a grid deformation which can be kept static throughout a simulation. In that case, Pulay force corrections, grid regeneration, and orbital interpolation will not be required. Such an approach is similar to that of Hamann. ${ }^{87}$

Another point to note from Fig. 1 is that when the Gaussian factor in Eq. (26) becomes sufficiently small, i.e., away from the atoms, the curvilinear coordinates reduce to the Cartesian coordinates. In these regions of space, the finite
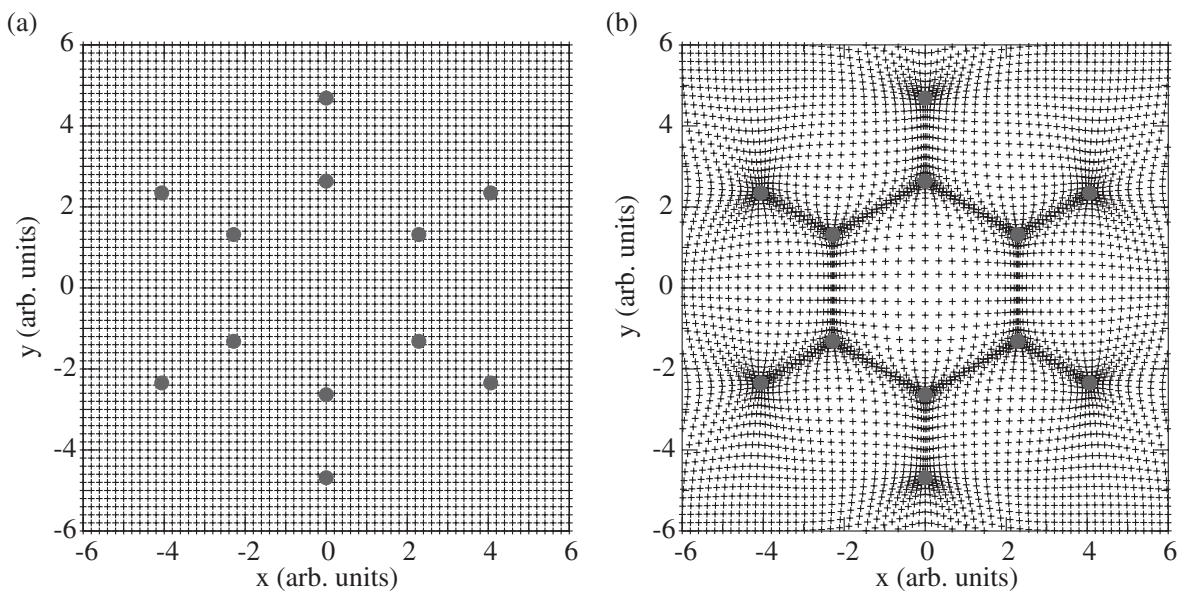

FIG. 1. Illustration of local adaptation around the 12 atoms in the benzene molecule (denoted by the grey circles) using the transformation between Cartesian and curvilinear coordinates described by Eq. (26). The molecule lies in the $x-y$ plane and for clarity only two dimensions are shown. (a) Shows the Cartesian grid under no adaptation, while (b) shows the deformation of the grid when adaptation is present. 
difference grid will become independent of the ion positions and those terms in Eqs. (16) and (20) depending on derivatives of the electronic coordinates with respect to the ionic coordinates will vanish.

\section{The Kohn-Sham equations in adaptive curvilinear coordinates}

With these coordinate transformations we can rewrite the time-dependent Kohn-Sham equations in terms of the curvilinear coordinates, $\zeta$. Referring to the Lagrangian in Eq. (15) and the Kohn-Sham equations of Eq. (16) we require expressions for the volume element and the Laplacian operator. Integrals will transform according to

$$
\int d \boldsymbol{r} f(\boldsymbol{r}) \rightarrow \int|J| d \zeta f(\zeta)
$$

while the Laplacian is given by the Lapace-Beltrami operator

$$
\nabla^{2}=\frac{1}{|J|} \frac{\partial}{\partial \zeta^{\alpha}}|J| g^{\alpha \beta} \frac{\partial}{\partial \zeta^{\beta}},
$$

where $g^{i j}$ is the contravariant metric tensor and Einstein summation notation is assumed. Transforming the Kohn Sham orbitals according to

$$
\psi_{i \sigma}(\boldsymbol{r}, t)=|J|^{-1 / 2} \varphi_{i \sigma}(\boldsymbol{r}, t),
$$

results in the Laplacian operator in the Kohn-Sham equations taking the form

$$
\nabla^{2}=\frac{1}{\sqrt{|J|}} \frac{\partial}{\partial \zeta^{\alpha}}|J| g^{\alpha \beta} \frac{\partial}{\partial \zeta^{\beta}} \frac{1}{\sqrt{|J|}} .
$$

This is important when a finite difference treatment of the Laplacian is used so that the resulting finite difference equations remain symmetric, thus allowing the use of unitary time propagation schemes for the solution of the Kohn-Sham equations. In three-dimensional space the Laplacian will have nine terms, three of which involve first derivatives in the same variable. These three terms can be rewritten so that they involve second derivatives in the same variable while still maintaining the symmetry of the finite difference Laplacian. For example, if we consider the Laplacian term involving $\zeta^{1}$ we can write

$$
\begin{aligned}
& \frac{1}{\sqrt{|J|}} \frac{\partial}{\partial \zeta^{1}}|J| g^{11} \frac{\partial}{\partial \zeta^{1}} \frac{1}{\sqrt{|J|}} \varphi_{i \sigma}(\boldsymbol{r}, t) \\
& =\frac{1}{2}\left[g^{11} \frac{\partial^{2}}{\partial \zeta^{12}}+\frac{\partial^{2}}{\partial \zeta^{12}} g^{11}\right] \varphi_{i \sigma}(\boldsymbol{r}, t)+M^{11} \varphi_{i \sigma}(\boldsymbol{r}, t),
\end{aligned}
$$

where

$$
\begin{aligned}
M^{11}= & \frac{1}{4|J|^{2}}\left[\left(|J|^{\prime}\right)^{2} g^{11}-2|J|^{2} g^{11^{\prime \prime}}\right. \\
& \left.-2|J||J|^{\prime} g^{11^{\prime}}-2|J||J|^{\prime \prime} g^{11}\right],
\end{aligned}
$$

and $f^{\prime}$ denotes differentiation of $f$ with respect to $\zeta^{1}$. As well as preserving the symmetry of the resulting finite difference equations, this symmetrization also reduces communication overheads when implemented as a parallel computer code us- ing domain decomposition. We shall discuss this further in Sec. V B.

\section{IMPLEMENTATION OF THE METHOD}

A typical calculation requires an initial ionic configuration to be chosen. Using this configuration the density is calculated self-consistently from the Kohn-Sham equations. This density and configuration is then used in the solution of equations of motion for the electrons and ions in the presence of a laser pulse. In this section we describe the technical details of our real-space implementation of the NAQMD approach in adaptive curvilinear coordinates.

\section{A. Discretization of the Kohn-Sham equations using finite differences}

The curvilinear coordinates $\zeta=\left(\zeta^{1}, \zeta^{2}, \zeta^{3}\right)$ are discretized on a finite difference grid with constant grid spacings $\left(\Delta \zeta^{1}, \Delta \zeta^{2}, \Delta \zeta^{3}\right)$ in each dimension. For coordinate $\zeta^{i}$, where $i=(1,2,3)$ we choose a set of $N_{\zeta^{i}}$ points which cover the range $-\zeta_{\max }^{i} \leq \zeta^{i} \leq \zeta_{\max }^{i}$ so that the grid spacing is given by

$$
\Delta \zeta^{i}=\frac{2 \zeta_{\max }^{i}}{N_{\zeta^{i}}-1}
$$

and the grid points are denoted by

$$
\zeta_{v}^{i}=-\zeta_{\max }^{i}+(v-1) \Delta \zeta^{i}, \quad v=1, \ldots, N_{\zeta^{i}}
$$

Derivatives appearing in the Laplacian - Eqs. (28) and (31) - are approximated by central difference formulae. The first derivative of a function, $f\left(\zeta^{i}\right)$, at the point $\zeta_{v}^{i}$ can be approximated by

$$
f^{\prime}\left(\zeta_{\nu}^{i}\right)=\frac{1}{\Delta \zeta^{i}} \sum_{\kappa=-N_{\mathrm{fd}}}^{N_{\mathrm{fd}}} C_{\kappa}^{(1)} f\left(\zeta_{\nu+\kappa}^{i}\right)
$$

while the second derivative is approximated as

$$
f^{\prime \prime}\left(\zeta_{\nu}^{1}\right)=\frac{1}{\left(\Delta \zeta^{i}\right)^{2}} \sum_{\kappa=-N_{\mathrm{fd}}}^{N_{\mathrm{fd}}} C_{\kappa}^{(2)} f\left(\zeta_{\nu+\kappa}^{i}\right)
$$

In these two equations $C_{\kappa}^{(1)}$ and $C_{\kappa}^{(2)}$ are the finite difference coefficients and the order of the finite difference formula is $2 N_{\mathrm{fd}}+1$. As a compromise between accuracy and sparsity in the resulting finite-difference equations, we generally use 5-point formulae, but higher-order formulae (up to 13-point) are also implemented.

Integrals of a function, as defined in Eq. (27), are simply approximated as

$$
\int d \boldsymbol{r} f(\boldsymbol{r}) \approx \Delta \zeta^{1} \Delta \zeta^{2} \Delta \zeta^{3} \sum_{\nu_{1}=1}^{N_{\zeta^{1}}} \sum_{\nu_{2}=1}^{N_{\zeta^{2}}} \sum_{\nu_{3}=1}^{N_{\zeta^{3}}}|J| f\left(\zeta_{\nu_{1}}^{1}, \zeta_{\nu_{2}}^{2}, \zeta_{\nu_{3}}^{3}\right)
$$




\section{B. Parallelization}

The parallel solution of the Kohn-Sham equations is achieved by a domain decomposition in which the full finitedifference grid is distributed over processors, with each processor storing all Kohn-Sham orbitals for that spatial region. Such a decomposition allows for greater scalability of the code compared to a parallelization over Kohn-Sham orbitals. This is particularly important when studying the interaction of molecules with intense laser pulses where we must use large spatial grids to hold the ionizing wavepackets. As we will see below, a grid parallelization will result in communication patterns that only involves halo grid points. A parallelization over Kohn-Sham orbitals, on the other hand, would suffer from two major drawbacks. First, for operations involving different Kohn-Sham orbitals, such as calculation of the density, the whole orbital must be transferred. This results in a large communication overhead. Second, a given system will only have a finite, potentially small, number of Kohn-Sham orbitals. This places a limit on the maximum number of processors that can be used in a given calculation.

A full decomposition of the finite difference grid is carried out in each spatial dimension. In a particular calculation we use $N_{p}^{i}$ processors in the $\zeta^{i}$ direction, where $i=(1,2$, 3), so that the calculation uses $N_{p}=N_{p}^{1} N_{p}^{2} N_{p}^{3}$ processors in total. Each processor in the $\zeta^{i}$ direction is labeled as

$$
P_{\rho}^{i}=\rho \quad \rho=0, \ldots, N_{p}^{i} .
$$

On each processor we have $N_{\text {loc }}^{i}$ local $\zeta^{i}$-points along the $\zeta^{i}$ direction so that $N_{\zeta^{i}}=N_{\mathrm{loc}}^{i} N_{p}^{i}$. Each local point on processor $P_{\rho}^{i}$ in the $\zeta^{i}$ direction can be mapped to a global point defined in Eq. (34) by

$$
\zeta_{v}^{i}=-\zeta_{\max }^{i}+\left(N_{\mathrm{loc}}^{i} \times P_{\rho}^{i}+\mu-1\right) \times \Delta \zeta^{i},
$$

where $\mu=1, \ldots, N_{\text {loc }}^{i}$.

The main communications bottleneck using this decomposition takes place when applying the Laplacian operator. A typical communications pattern for applying the Laplacian is presented in Fig. 2. For clarity we will limit our discussion to 2D and consider the case of using a 5-point approximation of both first and second derivatives appearing in Eqs. (30) and (31). In this figure the circles denote the points held on one processor, while the squares and triangles denote information required from other processors. When orthogonal curvilinear coordinates are used the information denoted by the triangles is not required and thus the use of nonorthogonal coordinate transformations (such as that described in Sec. IV B) increases the communication overhead in a calculation. The benefit of using Eq. (31) for evaluating those terms in the Laplacian involving derivatives in the same variable is now apparent. With Eq. (31) only two halo points are required for communication on each processor boundary. Alternatively, if we use the first derivative finite difference expressions directly in Eq. (30), this would entail extra communication overhead. For example, the half point rule suggested by Modine et al. ${ }^{73}$ would result in three halo points being required on each processor boundary.

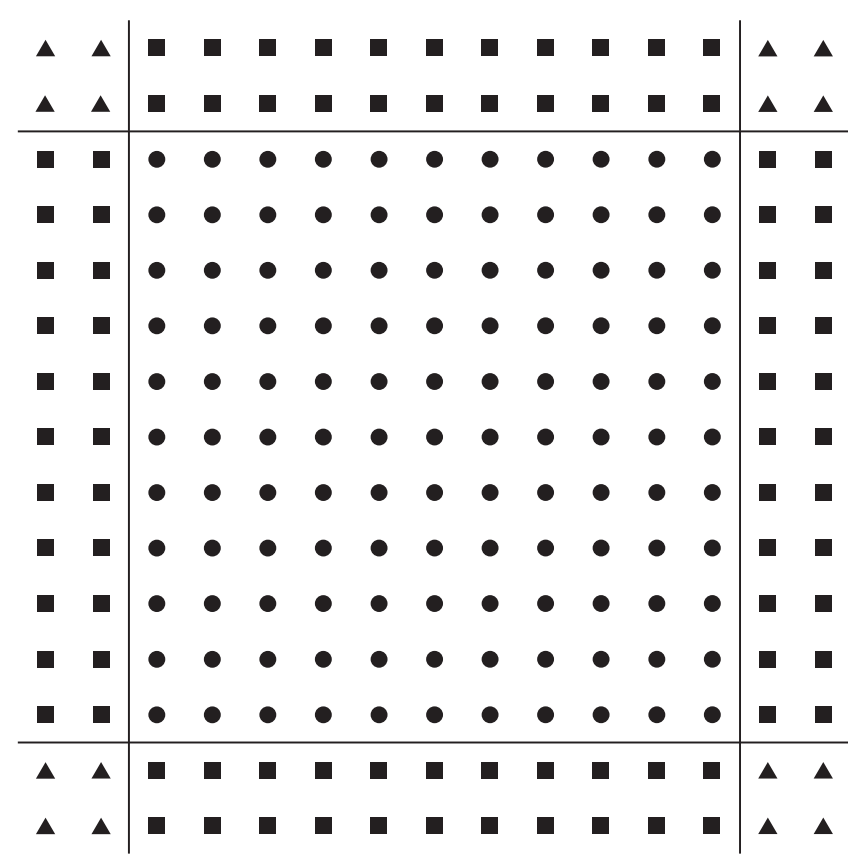

FIG. 2. Schematic diagram of communication pattern required for application of the Laplacian given in Eqs. (30) and (31) for a 3D domain decomposition of the finite difference grid. Processor boundaries are represented by the solid lines and for clarity only two dimensions are shown. The grid points local to a given processor are represented by $(\boldsymbol{O})$ while the halo points required from other processors are denoted by $(\boldsymbol{\square})$ and $(\boldsymbol{\Delta})$. Halo points denoted by $(\boldsymbol{\Delta})$ are required for application of those terms in the Laplacian in Eq. (30) that involve derivatives in different variables when using non-orthogonal curvilinear coordinates. Therefore, the use of non-orthogonal coordinates introduces an increased communications overhead in a given calculation.

\section{Time propagation}

To propagate the solutions of the NAQMD equations in time we must handle both electrons, Eq. (16), and ions, Eq. (20). Since the ions move more slowly than the electrons, we can use different time steps for each of these subsystems. In the following we denote the time step for the evolution of the electronic subsystem by $\Delta t_{e}$ while the time step for the ionic subsystem is denoted by $\Delta t_{i}$.

\section{Time propagation of the Kohn-Sham equations}

Time-propagation of the Kohn-Sham equations requires the use of a unitary propagator. Given a Kohn-Sham orbital $\varphi_{i \sigma}(\boldsymbol{r}, t)$ at a time $t$, the orbital at a later time, $t+\Delta t_{e}$, is obtained by applying the unitary time evolution operator $U_{i \sigma}\left(t+\Delta t_{e}, t\right)$, namely,

$$
\begin{aligned}
\varphi_{i \sigma}\left(\boldsymbol{r}, t+\Delta t_{e}\right) & =U_{i \sigma}\left(t+\Delta t_{e}, t\right) \varphi_{i \sigma}(\boldsymbol{r}, t) \\
& \approx e^{-\mathrm{i} H_{\mathrm{ks}, \sigma}(t) \Delta t_{e}} \varphi_{i \sigma}(\boldsymbol{r}, t) .
\end{aligned}
$$

We use the accurate, high-order unitary $n$ th-order Arnoldi propagator to approximate the evolution operator $U_{i \sigma}\left(t+\Delta t_{e}, t\right){ }^{63,88,89}$

\section{Time propagation of the ionic equations of motion}

For time-propagation of the classical classical equations of motion we use a velocity Verlet algorithm. In that case we 
evolve the positions and velocities of ion $k$ according to

$$
\begin{aligned}
& \boldsymbol{R}_{k}\left(t+\Delta t_{i}\right)=\boldsymbol{R}_{k}(t)+\dot{\boldsymbol{R}}_{k}(t) \Delta t_{i}+\frac{1}{2} \ddot{\boldsymbol{R}}_{k}(t)\left(\Delta t_{i}\right)^{2}, \\
& \dot{\boldsymbol{R}}_{k}\left(t+\Delta t_{i}\right)=\dot{\boldsymbol{R}}_{k}(t)+\frac{\ddot{\boldsymbol{R}}_{k}(t)+\ddot{\boldsymbol{R}}_{k}\left(t+\Delta t_{i}\right)}{2} \Delta t_{i},
\end{aligned}
$$

where $\ddot{\boldsymbol{R}}_{k}(t)$ is given by Eq. (20).

\section{Calculation of the initial state}

Before time propagation of the NAQMD equations we need to calculate the initial state of the system. We will consider the system to be in its ground electronic state for a given ionic configuration. In that case only the ground-state electronic density is required. ${ }^{58}$ If we require both the electronic and ionic system to be in equilibrium, this can be achieved by an iterative procedure in which the ionic configuration is optimized to minimize the ground-state energy of the system. For a given geometry, the ground-state energy is obtained from solving the time-independent Kohn-Sham equations self-consistently. The limited memory BFGS method ${ }^{90}$ is then used to find a better approximation to the equilibrium geometry. This procedure is repeated until convergence to a desired tolerance is achieved.

The major bottleneck in this procedure is the selfconsistent solution of the Kohn-Sham equations at each step of the optimization procedure. The method for self-consistent solution of the time-independent Kohn-Sham equations is set out in the following six steps.

1. Get initial guess for Kohn-Sham orbitals, $\varphi_{i \sigma}(\boldsymbol{r})$

2. Calculate charge density $n(\boldsymbol{r})$

3. Calculate effective potential $V_{\mathrm{eff}, \sigma}(\boldsymbol{r})$

4. Solve Kohn-Sham equations

$$
\left[\frac{1}{2} \nabla^{2}+V_{\mathrm{eff}, \sigma}(\boldsymbol{r})\right] \varphi_{i \sigma}(\boldsymbol{r})=\epsilon_{i} \varphi_{i \sigma}(\boldsymbol{r}), \quad i=1, \ldots
$$

5. Calculate new charge density, $\eta(\boldsymbol{r})$

6. If $|\eta(\boldsymbol{r})-n(\boldsymbol{r})|<\varepsilon$

$$
\begin{aligned}
& \text { Stop } \\
& \text { else } \\
& \text { Mix densities } \\
& \text { Calculate the new effective potential } V_{\text {eff, } \sigma}(\boldsymbol{r}) \\
& \text { Go to step } 4 \\
& \text { end }
\end{aligned}
$$

In the above scheme, $\varepsilon$ denotes the tolerance at which we decide the calculation has converged. While the convergence criteria outlined in this scheme involves the density, we have also implemented a convergence test based on the effective potential. We find that for sufficiently small values of $\varepsilon$, the results agree well and the computational time is similar. In addition, the density obtained through the solution of the Kohn-Sham equations is not used directly for the next iteration. Instead we carry out density mixing using either Anderson $^{91}$ or Pulay ${ }^{92}$ mixing.

The most time-consuming part of the self-consistent calculation is obtaining the Kohn-Sham eigenpairs at step 4. We require methods for calculating the ground-state density that are as efficient as possible and allow for effective parallelization. Several methods are now described.

\section{Propagation in imaginary time}

The Kohn-Sham eigenenergies and eigenvectors can be obtained through propagating the time-dependent KohnSham equations in imaginary time, subject to the constraint that the orbitals maintain orthogonality. This is achieved by making the replacement $\Delta \tau=\mathrm{i} \Delta t_{e}$ in Eq. (40). This has the effect of turning the Kohn-Sham equations into a set of diffusion equations, in which the eigenvectors decay at rates proportional to their energies. Such an approach has been used by several groups. ${ }^{63,93}$

\section{Thick-restarted Lanczos (TRLan) method}

Lanczos $^{94}$ showed that the eigen-decomposition of the Krylov-subspace Hamiltonian can be used as a first step in an iterative scheme to calculate the eigenvalues of the actual Hamiltonian. This approach can prove more attractive than time-propagation in imaginary time, especially when many eigenpairs are required. However, in this case, the Lanczos method becomes computationally expensive due to the size of the Krylov subspace required and the cost of maintaining orthogonality of the subspace vectors. A number of schemes have been proposed to reduce this cost ranging from partially reorthogonalized schemes ${ }^{95}$ which aim to reduce the computational cost associated with maintaining orthogonality of the Krylov vectors to restarted methods ${ }^{96}$ which aim to reduce the dimension of the Krylov subspace.

We have implemented the thick restart Lanczos method in the TRLan library ${ }^{96}$ in order to calculate the eigenenergies and eigenvectors of the required Kohn-Sham orbitals.

\section{The Chebyshev filtered subspace iteration (CheFSI) method}

As stated earlier, the major bottleneck in the calculation of the ground-state is the calculation of the Kohn-Sham eigenpairs in each self-consistent cycle. Recently, Zhou et al. ${ }^{97,98}$ developed an approach that emphasises the importance of eigenspaces rather than eigenpairs in the self-consistent solution of the Kohn-Sham equations. The basis of their approach is the fact that the density can be calculated from diagonal of the density matrix

$$
\Pi=\Phi \Phi^{\dagger},
$$

where $\Phi$ is the matrix whose columns are the occupied KohnSham orbitals. For any unitary matrix, $U$, of appropriate dimension we can write

$$
\Pi=(\Phi U)(\Phi U)^{\dagger},
$$

and thus explicit eigenvectors are not required in order to calculate the density, instead any orthonormal basis of the eigenspace corresponding to the occupied Kohn-Sham states will do. In their approach, the eigenproblem is solved once in order to provide an approximate eigenspace. This eigenspace 
must only be slightly larger than the number of states required. The approximate eigenspace is then filtered in each cycle of the self-consistency loop using a Chebyshev filter, $p_{m}(x)$, of order $m$ constructed from the Hamiltonian, $H_{\mathrm{ks},} \sigma$. The application of this filter on the eigenspace, $p_{m}\left(H_{\mathrm{ks}, \sigma}\right) \Phi$, should then provide a better approximation to the eigenspace of occupied states.

The filter is based upon the fast-growth property of Chebyshev polynomials of the first kind outside the interval $[-1,1]$. It is constructed by obtaining estimates for the lower and upper bound of the unwanted part of the spectrum of $H_{\mathrm{ks}, \sigma}$. These bounds can easily be calculated: see Zhou et al. ${ }^{97}$ for details. The filter is then constructed so that the unwanted part of the spectrum is mapped onto the region $[-1,1]$. In that case, the self-consistency loop becomes

1. Get initial guess for Kohn-Sham orbitals, $\varphi_{i \sigma}(\boldsymbol{r})$

2. Calculate charge density $n(\boldsymbol{r})$

3. Calculate the effective potential $V_{\text {eff, } \sigma}(\boldsymbol{r})$

4. Solve Kohn-Sham equations

$$
\left[\frac{1}{2} \nabla^{2}+V_{\mathrm{eff}, \sigma}(\boldsymbol{r})\right] \varphi_{i \sigma}(\boldsymbol{r})=\epsilon_{i} \varphi_{i \sigma}(\boldsymbol{r}), \quad i=1, \ldots
$$

5. Calculate new charge density, $\eta(\boldsymbol{r})$

6. If $|\eta(\boldsymbol{r})-n(\boldsymbol{r})|<$ tol

$$
\text { else }
$$

Mix densities

Calculate the new effective potential $V_{\text {eff }, \sigma}(r)$

Perform Chebyshev subspace iteration

Go to step 5

end

At step 4 any appropriate eigensolver can be used to provide an initial approximation to the eigenspace: we use the TRLan package.

\section{E. Calculation of the Hartree potential}

The Hartree potential integral in Eq. (19) is evaluated by solving the corresponding Poisson equation

$$
\nabla^{2} V_{H}(\boldsymbol{r}, t)=-4 \pi n(\boldsymbol{r}, t)
$$

Writing $V_{H}(\boldsymbol{r}, t)=|J|^{-1 / 2} W_{H}(\boldsymbol{r}, t)$, as in Eq. (29), results in the Laplacian in the Poisson equation having the form given by Eq. (30). Thus,

$$
\frac{1}{\sqrt{|J|}} \frac{\partial}{\partial \zeta^{\alpha}}|J| g^{\alpha \beta} \frac{\partial}{\partial \zeta^{\beta}} \frac{1}{\sqrt{|J|}} W_{H}(\boldsymbol{r}, t)=-4 \pi|J|^{1 / 2} n(r, t) .
$$

This equation is solved using a conjugate gradient method on our finite difference grid. In order to apply the correct boundary conditions we consider a multipole expansion of the
Hartree potential on the boundary of the grid so that

$$
\left.n(\boldsymbol{r})\right|_{\text {boundary }}=\sum_{l=0}^{\infty} \sum_{m=-l}^{l} \frac{4 \pi}{2 l+1} \frac{1}{r^{l+1}} Y_{l m}(\hat{\boldsymbol{r}}) Q_{l m},
$$

where

$$
Q_{l m}=\int d \boldsymbol{r} r^{l} n(\boldsymbol{r}) Y_{l m}^{\star}(\hat{\boldsymbol{r}}),
$$

are the multipole moments of the density.

\section{F. Treatment of the exchange-correlation potential}

All many-body effects are included within the exchangecorrelation potential, which in practice must be approximated. While many sophisticated approximations to this potential have been developed, ${ }^{99}$ the simplest is the adiabatic local density approximation (LDA) in the exchange-only limit (xLDA). In this case the exchange energy functional is given by

$$
E_{x}[n]=-\frac{3}{2}\left(\frac{3}{4 \pi}\right)^{1 / 3} \sum_{\sigma=\downarrow, \uparrow} \int d \boldsymbol{r} n_{\sigma}^{4 / 3}(\boldsymbol{r}, t),
$$

from which the exchange-correlation potential

$$
V_{\mathrm{xc}, \sigma}(\boldsymbol{r}, t)=-\left(\frac{6}{\pi}\right)^{1 / 3} n_{\sigma}^{1 / 3}(\boldsymbol{r}, t),
$$

can be obtained. While this approximate functional is easy to implement it does suffer from the drawback of containing self-interaction errors. This self-interaction means that the asymptotic form of the potential is exponential instead of Coulombic and many of the ground-state properties of atoms and molecules can differ significantly from experimental values, as we shall see in Sec. VI A 2. In spite of this problem, the LDA is one of the most widely used exchange-correlation functionals and will be used in this work in order to compare with previously published results.

\section{G. Treatment of the electron-ion potentials}

The Coulomb potential entering the external potential in the Kohn-Sham Hamiltonian, $V_{\text {ions }}(\boldsymbol{r}, \boldsymbol{R})$ as defined in Eq. (6) with $\boldsymbol{r}_{i}$ replaced by $\boldsymbol{r}$, is singular whenever a finite-difference grid point coincides with one of the ions. Two approaches can be used to deal with these singularities depending on whether we wish to carry out all-electron calculations or if we only need to describe the response of valence electrons.

\section{All-electron calculations}

If we want to carry out simulations in which the response of all electrons must be included, for instance in simulating the response of molecules to XUV laser pulses, then we require an approach that accurately approximates the Coulomb potential associated with each ion. At the same time any singularities that arise must be removed. We follow the approach of Modine et al. ${ }^{73}$ who calculate the Coulomb potential associated with each ion as the solution of a Poisson equation which approximates the volume charge density associated with a point charge. In that case, a point charge carrying 
charge $Z_{k}$ located at the point $\boldsymbol{R}_{k}$ may be described by the volume charge distribution $Z_{k} \delta\left(\boldsymbol{r}-\boldsymbol{R}_{k}\right)$, where $\delta(\boldsymbol{x})$ is the Dirac delta function. We approximate the Dirac delta function as

$$
\delta\left(\boldsymbol{r}-\boldsymbol{R}_{k}\right) \approx A_{k} \exp \left[-\frac{\left(\boldsymbol{r}-\boldsymbol{\Theta}_{k}\right)^{2}}{\gamma_{k}^{2}}\right]
$$

for $\gamma_{k}>0$. In this equation $A_{k}$ is a normalization factor and $\boldsymbol{\Theta}_{k}$ is a fictitious ion position. Both these parameters are chosen to satisfy

$$
\begin{gathered}
\int d \boldsymbol{r} A_{k} \exp \left[-\frac{\left(\boldsymbol{r}-\boldsymbol{\Theta}_{k}\right)^{2}}{\gamma_{k}^{2}}\right]=1, \\
\int d \boldsymbol{r} \boldsymbol{r} A_{k} \exp \left[-\frac{\left(\boldsymbol{r}-\boldsymbol{\Theta}_{k}\right)^{2}}{\gamma_{k}^{2}}\right]=\boldsymbol{R}_{k} .
\end{gathered}
$$

As in Sec. IV B, $\boldsymbol{\Theta}_{k} \neq \boldsymbol{R}_{k}$ in general to allow for situations in which different locally adapted regions overlap.

Solution of the Poisson equation,

$$
\nabla^{2} V_{k}^{D}\left(\boldsymbol{r}-\boldsymbol{R}_{k}\right)=4 \pi Z_{k} A_{k} \exp \left[-\frac{\left(\boldsymbol{r}-\boldsymbol{\Theta}_{k}\right)^{2}}{\gamma_{k}^{2}}\right],
$$

for ion $k$ then gives an approximation, $V_{k}^{D}\left(\boldsymbol{r}-\boldsymbol{R}_{k}\right)$, to the Coulomb potential so that

$$
V_{\text {ions }}(\boldsymbol{r}, \boldsymbol{R}) \approx \sum_{k=1}^{N_{n}} V_{k}^{D}\left(\boldsymbol{r}-\boldsymbol{R}_{k}\right) .
$$

The force acting of ion $k$ due to this potential will then be given by

$$
\begin{aligned}
\boldsymbol{F}_{k} & =-\int d \boldsymbol{r} n(\boldsymbol{r}) \tilde{\nabla}_{k} V_{k}^{D}\left(\boldsymbol{x}_{k}\right) \\
& =\int d \boldsymbol{r} n(\boldsymbol{r}) \nabla V_{k}^{D}\left(\boldsymbol{x}_{k}\right),
\end{aligned}
$$

where $\boldsymbol{x}_{k}=\boldsymbol{r}-\boldsymbol{R}_{k}$.

\section{Pseudopotential calculations}

To describe the interaction of molecules with laser pulses operating at Ti:sapphire wavelengths, we expect the laser to couple predominantly to valence electrons. In that case allelectron calculations are not necessary and we can replace the Coulomb potentials with pseudopotentials. We implement norm-conserving Troullier-Martins pseudopotentials ${ }^{100}$ in their fully separable Kleinman-Bylander form ${ }^{101}$

$$
V_{\text {ions }}(\boldsymbol{r}, \boldsymbol{R})=\sum_{k=1}^{N_{n}}\left[V_{k, \mathrm{ps}}^{l_{\mathrm{loc}}}\left(\boldsymbol{x}_{k}\right)+\sum_{\substack{l \\ l \neq l_{\mathrm{loc}}}} \sum_{m=-l}^{l} \frac{\left|\Delta V_{k, \mathrm{ps}}^{l}\left(\boldsymbol{x}_{k}\right) \chi_{l m}^{k}\left(\boldsymbol{x}_{k}\right)\right\rangle\left\langle\Delta V_{k, \mathrm{ps}}^{l}\left(\boldsymbol{x}_{k}\right) \chi_{l m}^{k}\left(\boldsymbol{x}_{k}\right)\right|}{\left\langle\chi_{l m}^{k}\left(\boldsymbol{x}_{k}\right)\left|\Delta V_{k, \mathrm{ps}}^{l}\left(\boldsymbol{x}_{k}\right)\right| \chi_{l m}^{k}\left(\boldsymbol{x}_{k}\right)\right\rangle}\right],
$$

where $V_{k, \mathrm{ps}}^{l_{\text {loc }}}\left(\boldsymbol{x}_{k}\right)$ defines the local component of the pseudopotential of ion $k, \chi_{l m}^{k}\left(\boldsymbol{x}_{k}\right)$ denotes the pseudo-wavefunction for the partial wave $|\operatorname{lm}\rangle$ and

$$
\Delta V_{k, \mathrm{ps}}^{l}\left(\boldsymbol{x}_{k}\right)=V_{k, \mathrm{ps}}^{l}\left(\boldsymbol{x}_{k}\right)-V_{k, \mathrm{ps}}^{l_{\mathrm{loc}}}\left(\boldsymbol{x}_{k}\right),
$$

define the non-local components of the pseudopotential.

The force acting on ion $k$ due to this non-local pseudopotential is given by ${ }^{102}$

$$
\begin{aligned}
\boldsymbol{F}_{k}= & \int d \boldsymbol{r} n(\boldsymbol{r}) \nabla V_{k, \mathrm{ps}}^{l_{\mathrm{loc}}}\left(\boldsymbol{x}_{k}\right) \\
& +\sum_{\sigma=\downarrow, \uparrow} \sum_{i=1}^{N_{\sigma}} \sum_{\substack{l \\
l \neq l_{\mathrm{loc}}}} \sum_{m=-l}^{l}\left(\frac{B_{l m}^{i \sigma k}}{A_{l m}^{k}}\right) \nabla B_{l m}^{i \sigma k},
\end{aligned}
$$

where

$$
\begin{aligned}
& A_{l m}^{k}=\left\langle\chi_{l m}^{k}\left(\boldsymbol{x}_{k}\right)\left|\Delta V_{k, \mathrm{ps}}^{1}\left(\boldsymbol{x}_{k}\right)\right| \chi_{l m}^{k}\left(\boldsymbol{x}_{k}\right)\right\rangle, \\
& B_{l m}^{i \sigma k}=\left\langle\Delta V_{k, \mathrm{ps}}^{1}\left(\boldsymbol{x}_{k}\right) \chi_{l m}^{k}\left(\boldsymbol{x}_{k}\right) \mid \varphi_{i \sigma}(\boldsymbol{r}, t)\right\rangle .
\end{aligned}
$$

In this paper, all pseudopotentials were generated using the atomic pseudopotential engine. ${ }^{103}$

\section{H. Treatment of the laser-electron interaction}

Assuming that the dipole approximation is valid, we define the vector potential of the laser pulse to be

$$
\boldsymbol{A}(t)=A(t) \hat{\boldsymbol{e}}
$$

where $\hat{\boldsymbol{e}}$ is the polarization direction and

$$
A(t)=A_{0} f(t) \cos \left(\omega_{L} t+\phi\right),
$$

and where $\omega_{L}$ is the laser frequency, $\phi$ is the carrier-envelope phase and $f(t)$ the pulse envelope given by

$$
f(t)=\left\{\begin{array}{ll}
\sin ^{2}\left(\frac{\pi t}{T}\right) & 0 \leq t \leq T \\
0 & \text { otherwise }
\end{array},\right.
$$

for a pulse of duration $T$. For this choice of the vector potential, the electric field is given by $\boldsymbol{E}(t)=E(t) \hat{\boldsymbol{e}}$ where

$$
E(t)=E_{0} f(t) \sin \left(\omega_{L} t+\phi\right)-\frac{E_{0}}{\omega_{L}} \frac{\partial f}{\partial t} \cos \left(\omega_{L} t+\phi\right),
$$

and the peak electric field strength is related to the peak laser intensity, $I_{0}$, by

$$
E_{0}=\left(\frac{4 \pi I_{0}}{c}\right)^{1 / 2}
$$


The laser-electron interaction, $U_{\text {elec }}(\boldsymbol{r}, t)$, can be represented in several ways. In this work a length gauge description of the interaction is used, in which case

$$
U_{\text {elec }}(\boldsymbol{r}, t)=U_{L}(\boldsymbol{r}, t)=\boldsymbol{r} \cdot \boldsymbol{E}(t) .
$$

A velocity gauge description of the electron-field interaction can also be used but in previous grid calculations we have not observed any major differences in the results for these two gauges. ${ }^{63}$

\section{Wavefunction splitting}

Ionizing wavepackets which reach the edge of the finite difference grid can be reflected from the boundary causing spurious effects in both harmonic spectra and ionization rates. We eliminate these reflections by a splitting technique akin to an absorbing boundary which partitions the Kohn-Sham orbitals into two parts, one near, and the other far from the molecule where the Coulomb potential is negligible.$^{63,89}$ The splitting is implemented by a mask function, $M(\boldsymbol{r})$, which equals unity near the origin and goes asymptotically to zero very gradually. Using this mask function the Kohn-Sham orbital, $\varphi_{i \sigma}(\boldsymbol{r}, t)$, is split into two parts

$$
\varphi_{i \sigma}(\boldsymbol{r}, t)=M(\boldsymbol{r}) \varphi_{i \sigma}(\boldsymbol{r}, t)+\{1-M(\boldsymbol{r})\} \varphi_{i \sigma}(\boldsymbol{r}, t) .
$$

The residual part, $\{1-M(\boldsymbol{r})\} \varphi_{i \sigma}(\boldsymbol{r}, t)$, can be propagated independently in the limit in which the Coulomb potential is negligible over the region in which $M(r)<1$. The design and optimization of the mask function requires considerable care, and extensive numerical simulations must be performed to characterize the optimal shape. ${ }^{89}$

The mask function is written in the form

$$
M(\boldsymbol{r})=M_{x}(x) M_{y}(y) M_{z}(z),
$$

where $M_{x}(x)$ takes the form

$$
M_{x}(x)= \begin{cases}1 & |x| \leq x_{\text {split }} \\ \exp \left[-\left(\frac{x-x_{\text {start }}}{\theta_{x}}\right)^{2}\right] & x_{\text {split }}<|x| \leq x_{\max }\end{cases}
$$

In this equation

$$
\theta_{x}=\frac{x_{\text {max }}-x_{\text {split }}}{M_{\text {edge }}},
$$

where $M_{\text {edge }}=M_{x}\left(x_{\max }\right)$ denotes the value of $M_{x}(x)$ that we wish to impose at the boundary. Similar expressions are used to describe $M_{y}(y)$ and $M_{z}(z)$.

We note that the mask function does not have to equal zero at the boundary. The only requirement is that $\varphi_{i \sigma}(\boldsymbol{r}, t)$ smoothly approaches zero at the edges of the integration volume.

\section{RESULTS}

In this section we will use our mixed quantum-classical approach to study the influence of multielectron effects in HHG in molecules. Before presenting these results, we will compare the performance of the eigensolvers described in
Sec. V D. In addition, the accuracy of ground-state properties will be compared with results obtained using a different method and with experiment. Our HHG results will consider $\mathrm{N}_{2}$ and benzene interacting with linearly polarized laser pulses. We will show how symmetries in the Kohn-Sham orbitals can lead to either enhancement and suppression of the harmonics as the orientation between the laser and the molecule changes.

\section{A. Initial state generation: Accuracy and efficiency}

The first stage of any simulation is obtaining the initial state of the system. In Sec. V D three iterative approaches were described for calculating the ground-state of a given system. In general we find that propagation in imaginary time only performs efficiently when a small number of eigenpairs (less than five) are required. In light of this we only compare the thick-restarted Lanczos method with the Chebyshev filtered subspace iteration method. We then calculate ground state energies for a range of atoms and diatomic molecules and show that accurate results can be obtained using a set of transferable grid adaptation parameters.

\section{Efficiency of the eigensolvers}

In order to compare the accuracy of the eigensolvers we consider the calculation of the ground-state of $\mathrm{N}_{2}$ using a globally adapted grid. The grid is set up so that the parameters controlling its extent and grid spacing are similar to those that will be used in the HHG simulations later. We consider a grid that is globally adapted in the $x$ and $y$ directions using the transformation of Eq. (23), while the $z$ coordinate is left unscaled. The grid spacings in each direction is the same $\left(\Delta \zeta^{i}=0.4, i=1,2,3\right)$ and the number of grid points used in each direction was $N_{\zeta^{1}}=N_{\zeta^{2}}=57$ and $N_{\zeta^{3}}=1001$. With appropriate scaling parameters in $x$ and $y$ the final grid extent was $-120 \leq x \leq 120,-120 \leq y \leq 120$, and $-200 \leq z$ $\leq 200$. The calculation was parallelized with the $z$ coordinate being distributed over 15 processors. Troullier-Martins normconserving pseudopotentials were used for the electron-ion interactions.

In Table I we present results for the time taken to obtain the ground state energy for $\mathrm{N}_{2}$ : in all cases the convergence criteria used was that the least-squares norm of the difference in density between self-consistent cycles was less than $10^{-7}$. It can clearly be seen that the CheFSI method outperforms the TRLan method by a factor of 7 . For the TRLan results, the calculation time reduces as we go to higher order in the Lanczos method, however, this comes at the cost of a larger memory requirement. For the CheFSI method the lowerorder filter outperforms the higher-order filter. In this case the lower-order filter is sufficient for the number of eigenpairs required.

\section{Accuracy of the energy eigenstates using locally adaptive grids}

To show the accuracy of our approach in the calculation of the initial state when using locally adaptive 
TABLE I. Comparison of the efficiency of the TRLan and CheFSI eigensolvers in calculating the ground state of $\mathrm{N}_{2}$. The time taken to obtain a converged, accurate ground state is given for various Lanczos and Chebyshev filter orders. Other calculation parameters are detailed in the text.

\begin{tabular}{lcc}
\hline \hline Eigensolver & Order & Calculation time (s) \\
\hline TRLan & 18 & 8906 \\
& 30 & 7638 \\
CheFSI & 8 & 1051 \\
& 15 & 1528 \\
\hline \hline
\end{tabular}

grids, we have compared the static properties of a range of atoms and molecules with those obtained by Grabo et al. ${ }^{104}$ and with experiment. Table II presents results for atoms, Table III presents results for diatomic molecules, and Table IV presents results for benzene. For all calculations, we consider a grid that is locally adapted using the transformation given in Eq. (26). A 5-point finite difference rule was used and the grid spacings in each direction were kept the same $\left(\Delta \zeta^{i}=0.2, i=1,2,3\right)$. The number of grid points used was $N_{\zeta^{1}}=N_{\zeta^{2}}=N_{\zeta^{3}}=201$ so that the grid extent was -20 $\leq x \leq 20,-20 \leq y \leq 20$ and $-20 \leq z \leq 20$.

In these calculations all electrons are considered and the Coulomb potential associated with each ion calculated using Eq. (53). Appropriate grid adaptation and Coulomb potential parameters were chosen as follows. The atom with the largest charge is nitrogen. The parameters for this atom were chosen by tuning them to obtain the correct hydrogenic energy levels for nitrogen (by switching off the Hartree and exchange correlation potentials). The resulting parameters were then used for all other atoms and molecules. The results show that not only are accurate energies obtained (when compared with other xLDA calculations), but the adapted grid is transferrable to other atomic and molecular systems having smaller nuclear charge. However, it is clear that the properties calculated using the xLDA exchange-correlation functional dif-

TABLE II. xLDA all-electron atomic ground state energies and ionization potentials calculated using a locally adapted finite difference grid in 3D. The results of the present work are compared with experiment and with the theoretical calculations of Grabo et al. ${ }^{104}$ The current results agree well with the xLDA results of Grabo et al. Both ground state energies and ionization potentials are generally underestimated using xLDA. The ionization potentials, calculated as vertical ionization potentials, show much better agreement with experiment than the ground state energies. The grid adaptation and Coulomb potential parameters used were chosen to reproduce the correct hydrogenic energy levels of nitrogen: these parameters are used for all other species considered. Other calculation parameters are detailed in the text.

\begin{tabular}{lcccccc}
\hline \hline & \multicolumn{3}{c}{ Ground state energy } & & \multicolumn{2}{c}{ Ionization potential } \\
\cline { 2 - 4 } Atom & Present & Grabo $^{\mathrm{a}}$ & Expt. $^{\mathrm{a}}$ & & Present & Expt. $^{\mathrm{a}}$ \\
\hline $\mathrm{H}$ & -0.4568 & -0.4571 & -0.5000 & & 0.4568 & 0.5000 \\
$\mathrm{He}$ & -2.7191 & -2.7236 & -2.9037 & & 0.8442 & 0.9037 \\
$\mathrm{Li}$ & -7.1741 & -7.1934 & -7.4781 & & 0.1767 & 0.1982 \\
$\mathrm{Be}$ & -14.1834 & -14.2233 & -14.6674 & & 0.2835 & 0.3426 \\
$\mathrm{~B}$ & -24.0566 & -23.7791 & -24.6539 & & 0.2775 & 0.3050 \\
$\mathrm{C}$ & -37.0977 & -37.1119 & -37.8450 & & 0.3897 & 0.4140 \\
$\mathrm{~N}$ & -53.6854 & -53.7093 & -54.5893 & 0.4890 & 0.5348 \\
\hline \hline
\end{tabular}

${ }^{\mathrm{a}}$ Reference 104. fer significantly from experimental values. This is due to the limitations of the LDA as outlined in Sec. V F. Even so, the ionization potentials obtained from these calculations are in fairly good agreement with experiment. These have been calculated as vertical ionization potentials, rather than estimating them from the energy of the highest occupied molecular orbital (HOMO).

\section{B. Multielectron and orientation effects in molecular HHG}

We now consider HHG in molecules irradiated by intense, short-duration laser pulses. According to classical electromagnetism the emission of secondary radiation arises from accelerating dipole moments induced by the laser pulse. The emission of high-order harmonics is generally understood in terms of the three-step model of Corkum ${ }^{110}$ and Kulander and co-workers ${ }^{111}$ in which electrons initially tunnel through the field-modified Coulomb potential barrier, propagate in the laser field and eventually recombine with the ion. HHG spectra are calculated readily within TDDFT since only a simple functional of the electron density is involved. The spectral density is calculated from the Fourier transform of the dipole acceleration ${ }^{112}$

$$
S(\omega)=\left|\int d t e^{\mathrm{i} \omega t} \hat{\boldsymbol{e}} \cdot \ddot{\boldsymbol{d}}(t)\right|^{2} .
$$

The dipole acceleration, $\ddot{\boldsymbol{d}}(t)$, can be calculated via Ehrenfest's theorem as

$$
\ddot{\boldsymbol{d}}(t)=-\sum_{\sigma=\downarrow, \uparrow} \int n_{\sigma}(\boldsymbol{r}, t) \nabla V_{\mathrm{eff}, \sigma}(\boldsymbol{r}, \boldsymbol{R}, t) d \boldsymbol{r},
$$

where $V_{\text {eff, } \sigma}(\boldsymbol{r}, \boldsymbol{R}, t)$ is the effective potential given by Eq. (18). When using the LDA approximation, described in Sec. V F, it must be noted that the incorrect asymptotic behaviour of the exchange-correlation potential means that recollisions are not described with quantitative accuracy. ${ }^{47}$ However, the main qualitative features of HHG spectra are reproduced, as can be seen when LDA results are compared with asymptotically correct functionals. ${ }^{47}$

Using our approach we will now consider HHG when a linearly polarized laser pulse interacts with $\mathrm{N}_{2}$ and benzene. In all simulations the following parameters are used. We fix the laser polarization direction along either the $x$-axis or the $z$-axis. When the laser is aligned along the $z$-axis, we use a grid that is unadapted along the $z$-axis while the $x$-and $y$-axes are globally adapted using the transformation of Eq. (23). A 5-point finite difference rule will be used with equal grid spacings in each coordinate $\left(\Delta \zeta^{i}=0.4, i=1,2,3\right)$. The number of grid points used for each coordinate is $N_{\zeta^{1}}=N_{\zeta^{1}}=61$ and $N_{\zeta^{3}}=999$. With appropriate scaling parameters the final grid extent is $-120 \leq x \leq 120,-120 \leq y \leq 120$, and -199.6 $\leq z \leq 199.6$. In the case where the laser is aligned along the $x$-axis, the grid parameters for the $x$ - and $z$-axes are interchanged: we now globally adapt the grid along the $z$-axis while the grid remains unadapted along the $x$-axis and the extent of the grid in $x$ and $z$ becomes $-199.6 \leq x \leq 199.6$ and $-120 \leq z \leq 120$. Troullier-Martins norm-conserving pseudopotentials were used for the electron-ion interactions and 
TABLE III. xLDA all-electron equilibrium molecular bond lengths, dissociation energies, and ionization potentials using a locally adapted finite difference grid in 3D. The results of the present work are compared with experimental results. As in the atomic case, we see that ionization potentials are underestimated using xLDA. The grid adaptation and Coulomb potential parameters used were chosen to reproduce the correct hydrogenic energy levels in the nitrogen atom: these parameters are used for all other species considered. Other calculation parameters are detailed in the text.

\begin{tabular}{|c|c|c|c|c|c|c|}
\hline \multirow[b]{2}{*}{ Molecule } & \multicolumn{2}{|c|}{ Bond length, $R_{e}$} & \multicolumn{2}{|c|}{ Dissociation energy, $D_{e}$} & \multicolumn{2}{|c|}{ Ionization potential } \\
\hline & Present & Expt. $^{\mathrm{a}}$ & Present & Expt. $^{\mathrm{a}}$ & Present & Expt. $^{\mathrm{a}}$ \\
\hline $\mathrm{N}_{2}$ & 2.071 & 2.074 & 0.381 & 0.364 & 0.517 & 0.573 \\
\hline $\mathrm{LiH}$ & 3.120 & 3.015 & 0.080 & 0.092 & 0.267 & 0.283 \\
\hline $\mathrm{BH}$ & 2.414 & 2.329 & 0.148 & 0.135 & 0.308 & 0.359 \\
\hline
\end{tabular}

${ }^{\mathrm{a}}$ Reference 104.

time propagation was carried out using a 6th-order Arnoldi propagator with a time step $\Delta t_{e}=0.04$.

\section{Multielectron and orientation effects in $\mathrm{HHG}$ in $\mathrm{N}_{2}$}

Our calculations for $\mathrm{N}_{2}$ will consider its interaction with a 10-cycle linearly-polarized Ti-sapphire $(\lambda=780 \mathrm{~nm})$ laser pulse having a peak intensity of $4.0 \times 10^{14} \mathrm{~W} / \mathrm{cm}^{2}$. The internuclear spacing is set to $2.07 a_{0}$ and the molecule is aligned along the $z$-axis. For the two orientations of the laser considered, the polarization direction is either parallel (along the $z$-axis) or perpendicular (along the $x$-axis) to the molecular axis. All ions are kept fixed throughout the simulation. We consider the 5 valence Kohn-Sham orbitals in our simulations which are plotted in Fig. 3. Each orbital is labeled (a)-(e) as detailed in the figure. We can see that orbitals (c) and (d) will respond in a similar fashion to the field whenever the laser polarization is parallel to the molecular axis whereas they will respond differently whenever the laser polarisation is perpendicular to the molecular axis. It must be stressed at this point that the Kohn-Sham orbitals do not have any direct connection to the actual molecular orbitals. However, studying the evolution of the Kohn-Sham orbitals allows us to obtain information about the importance of orbital symmetries in the response and is widely used in TDDFT studies.

Figure 4 presents the HHG spectra. The cut-off in the plateau region of the spectrum is given by

$$
E_{\text {cut-off }}=1.32 I_{p}+3.17 U_{p},
$$

where $I_{p}$ is the ionization potential of the molecule and $U_{p}$ is the laser ponderomotive energy. ${ }^{113}$ Thus, for the laser parameters considered the cut-off should occur at harmonic 57, and indeed we see that our calculated cut-off agrees quite well with this value. For harmonic orders less than 21 the spectral density is greatest whenever the laser polarization is parallel to the molecular axis, while for higher harmonics the converse is true. Figure 5 presents the orbital populations during the interaction with the laser pulse perpendicular to the molecular axis. It is clear that the two forms of the $1 \pi_{u}$ molecular orbital (the HOMO-1 orbital) - (c) and (d) in Figure 3 - respond differently to the field and that the $1 \pi_{u}$ (d) orbital does indeed respond more than the HOMO. This is in contrast to the parallel orientation where both $1 \pi_{u}$ orbitals respond identically and where the HOMO responds predominantly. This type of behaviour is similar to that observed in ionization studies of OCS and $\mathrm{CS}_{2} \cdot{ }^{48}$ However, in previous DFT studies of $\mathrm{N}_{2}$ by Petretti et al., ${ }^{114}$ the $1 \pi_{u}$ orbital was observed to show a lower response than the HOMO in perpendicular alignment. These previous calculations were performed at a lower laser intensity and it is unclear if the two forms of the $1 \pi_{u}$ orbitals were included explicitly in their calculations.

We note that in a previous experiment, McFarland et al. $^{35}$ showed that in the perpendicular alignment the harmonic signal was enhanced, relative to the parallel case, around the cut-off in the plateau. This enhancement was attributed to the contribution from electrons in the more tightly bound $1 \pi_{u}$ HOMO- 1 orbital compared with electrons in the $3 \sigma_{g}$ HOMO. The influence of the HOMO-1

TABLE IV. xLDA all-electron static properties of benzene. The equilibrium $\mathrm{C}-\mathrm{C}$ and $\mathrm{C}-\mathrm{H}$ bond lengths, atomization energy and ionization potential are compared with experiment. We see that the atomization energy is greatly overestimated, in common with other LDA calculations. ${ }^{108,109}$ The grid adaptation and Coulomb potential parameters used were chosen to reproduce the correct hydrogenic energy levels in the nitrogen atom. Other calculation parameters are detailed in the text.

\begin{tabular}{|c|c|c|c|c|c|c|c|}
\hline \multicolumn{4}{|c|}{ Equilibrium geometry } & & & & \\
\hline \multicolumn{2}{|c|}{$\mathrm{C}-\mathrm{C}$ bond length } & \multicolumn{2}{|c|}{$\mathrm{C}-\mathrm{H}$ bond length } & \multicolumn{2}{|c|}{ Atomization energy } & \multicolumn{2}{|c|}{ Ionization potential } \\
\hline Present & Expt. $^{\mathrm{a}}$ & Present & Expt. $^{\mathrm{a}}$ & Present & Expt. $^{b}$ & Present & Expt. $^{c}$ \\
\hline 2.645 & 2.644 & 2.091 & 2.081 & 3.283 & 2.081 & 0.292 & 0.340 \\
\hline
\end{tabular}

${ }^{b}$ Reference 106

${ }^{\mathrm{c}}$ Reference 107. 
(a)

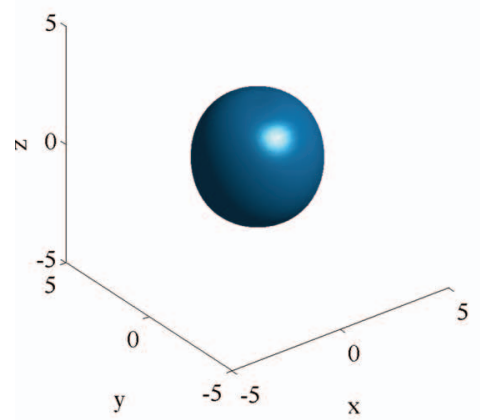

(d) (b)

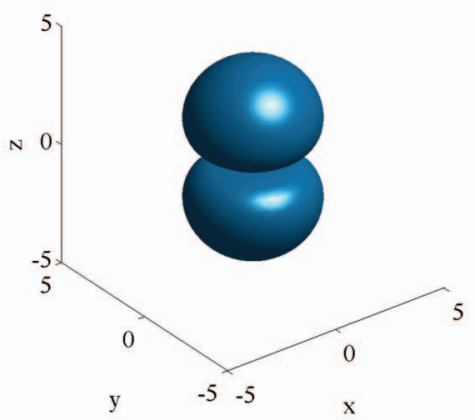

(c)

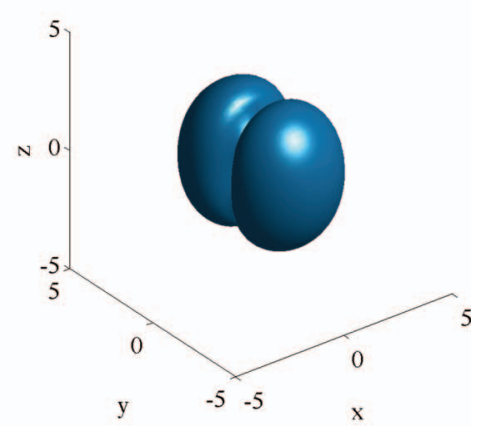

(e)
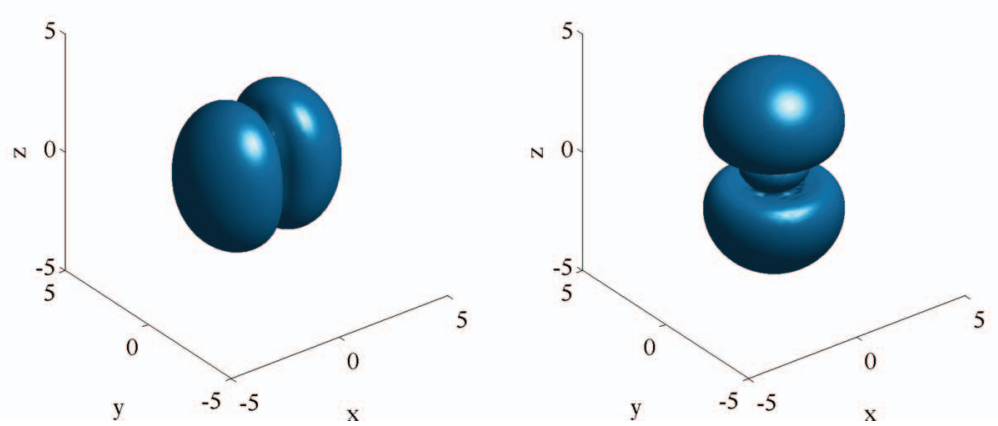

FIG. 3. Isosurface plots of the valence Kohn-Sham orbital densities in $\mathrm{N}_{2}$ considered in our calculations. The occupation of each Kohn-Sham orbital is 2 . The ground state energy configuration of $\mathrm{N}_{2}$ is $1 \sigma_{g}^{2} 1 \sigma_{u}^{2} 2 \sigma_{g}^{2} 2 \sigma_{u}^{2} 1 \pi_{u}^{4} 3 \sigma_{g}^{2}$ and so each orbital is labeled as follows: (a) $=2 \sigma_{g},(\mathrm{~b})=2 \sigma_{u}$, (c) $=1 \pi_{u}$, (d) $=1 \pi_{u}$, and (e) $=3 \sigma_{g}$. In these plots, the molecular axis is aligned along the $z$-axis and for the HHG results the laser polarization will either be along the $x$-or the $z$-axis.

orbital in $\mathrm{HHG}$ in the perpendicular orientation has also been studied in a more recent experiment. ${ }^{115}$ However, the results of McFarland et al. disagree with earlier experiments in which the harmonic signal was greater in the parallel orientation than in the perpendicular orientation. ${ }^{116-118}$ These earlier experiments were carried out at lower laser intensities and mainly considered plateau harmonics. It has been suggested that the results of McFarland et al. may

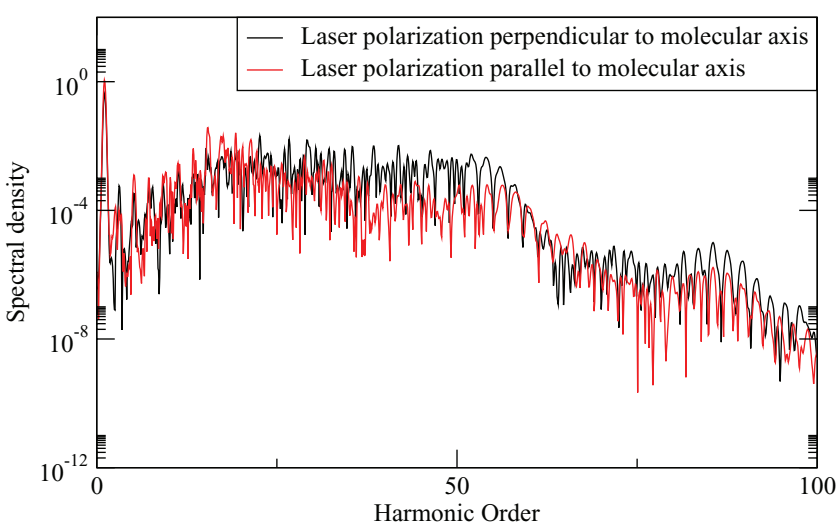

FIG. 4. HHG spectra for $\mathrm{N}_{2}$. The molecule interacts with a 10-cycle linearly polarized Ti-sapphire $(\lambda=780 \mathrm{~nm})$ laser pulse having a peak intensity of $4.0 \times 10^{14} \mathrm{~W} / \mathrm{cm}^{2}$. The molecular axis is aligned along the $z$-axis. The black line shows the spectrum when the laser polarization lies perpendicular to the molecular axis while the red line denotes the spectrum when the laser polarization lies parallel to the molecular axis. be due to propagation effects: ${ }^{119}$ these effects have not been considered in the present work. Additionally, in a range of calculations based on quantitative rescattering theory it has been found that the contribution of the HOMO1 orbital becomes important in the perpendicular alignment at high laser intensities when considering both the

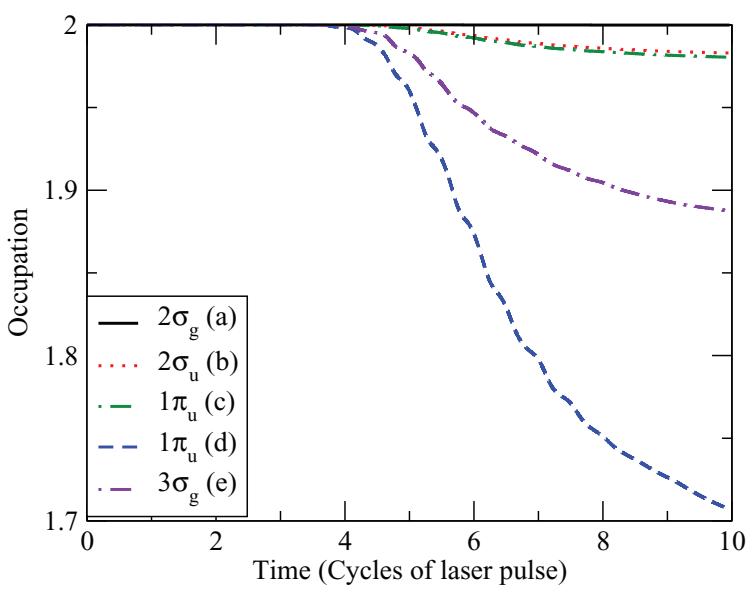

FIG. 5. Occupation of the valence Kohn-Sham orbitals of $\mathrm{N}_{2}$ during interaction with a 10 -cycle linearly polarized Ti-sapphire $(\lambda=780 \mathrm{~nm})$ laser pulse having a peak intensity of $4.0 \times 10^{14} \mathrm{~W} / \mathrm{cm}^{2}$. The laser polarization direction is perpendicular to the molecular axis. We see that the more tightly bound $1 \pi_{u}$ (d) orbital responds more than the $3 \sigma_{g}$ HOMO. The change in the occupation of the $2 \sigma_{g}$ orbital during the interaction with the pulse is too small to show up on this scale. 


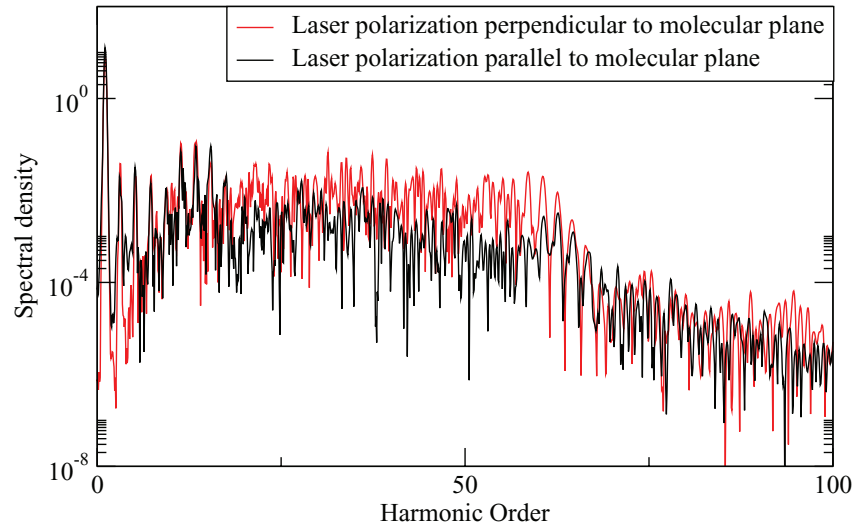

FIG. 6. HHG spectra for benzene. The molecule interacts with a 10-cycle linearly polarized Ti-sapphire $(\lambda=780 \mathrm{~nm})$ laser pulse having a peak intensity of $4.0 \times 10^{14} \mathrm{~W} / \mathrm{cm}^{2}$. The molecule lies in the $x-y$ plane as shown if Fig. 1. The red line shows the spectrum when the laser-polarization lies perpendicular to the molecular plane while the black line denotes the spectrum when the laser-polarization lies parallel to the plane of the molecule.

single atom response ${ }^{37}$ and when including propagation effects. $^{115}$

\section{Multielectron and orientation effects in HHG in benzene}

While most experimental studies of HHG in benzene consider the interaction with a linearly polarized laser pulse, ${ }^{30}$ theoretical studies generally study the response to circularly polarized light. ${ }^{31-33}$ In our simulations we will consider HHG using a 10-cycle linearly polarized Ti-sapphire $(\lambda=780 \mathrm{~nm})$ laser pulse having a peak intensity of $4.0 \times 10^{14} \mathrm{~W} / \mathrm{cm}^{2}$. The benzene molecule lies in the $x-y$ plane with the atom positions as shown in Fig. 1. For the two laser alignments the polarization direction is either parallel (along the $x$-axis) or perpendicular (along the $z$-axis) to the molecular plane. All ions are kept fixed throughout the simulation. Figure 6 presents harmonic spectra. For the laser parameters used, the cut-off region for the plateau, using Eq. (73), should occur around harmonic 53. We see that our results are in good agreement. The intensity of those harmonics of order less than 21 are comparable while for higher-order harmonics the harmonic intensity is greatest in the perpendicular orientation. The reason for the suppression in the parallel orientation can be understood by considering the symmetry of the HOMO orbital. In benzene, the HOMO is doubly degenerate: isosurface plots of these two orbitals are presented in Fig. 7 and are referred to as HOMO (a) and HOMO (b). Figure 8 presents the populations for the Kohn-Sham orbitals in the parallel orientation during the interaction with the pulse: for clarity we have only labeled the HOMO (a) and HOMO (b) orbitals. We see that the response of the HOMO (b) orbital is suppressed, relative to the HOMO (a). In the perpendicular alignment both HOMO orbitals respond in the same way to the field and the response is similar to that of the HOMO (a) in Fig. 8. This suggests that the reduction in the intensity of plateau harmonics near the cut-off in the parallel alignment is due to the symmetry-induced suppression of the HOMO (b) orbital.

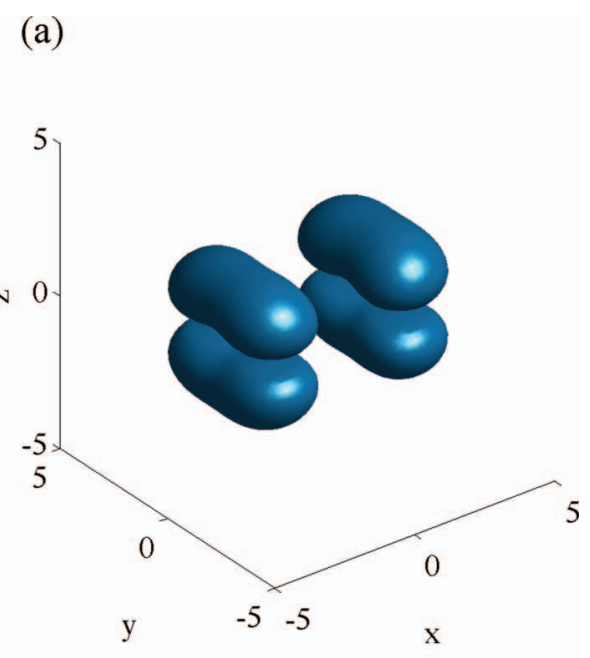

(b)

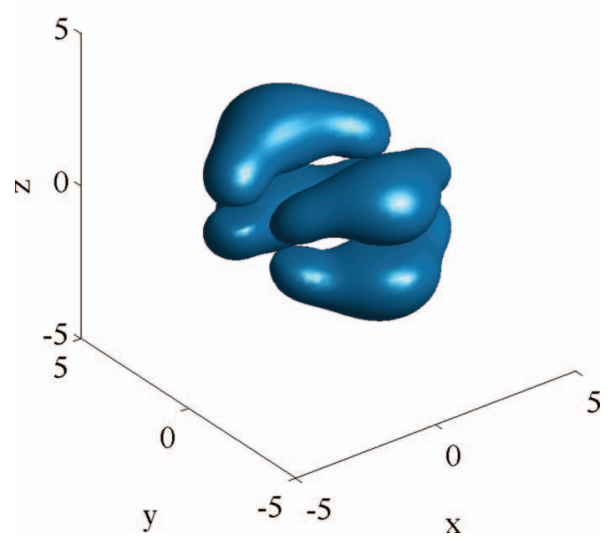

FIG. 7. Isosurface plots of the two degenerate HOMO Kohn-Sham orbital densities for benzene in our calculations. The molecule lies in the $x-y$ plane as denoted in Fig. 1. The occupation of each Kohn-Sham orbital is 2. We label these orbitals as HOMO (a) and HOMO (b). In these plots, the molecule lies in the $x-y$ plane and for the HHG results the laser polarization will either be along the $x$ - or the $z$-axis.

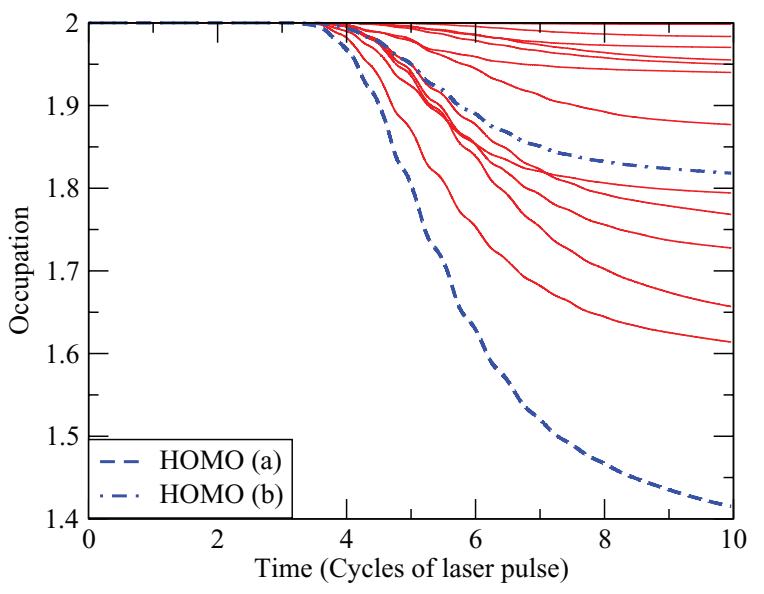

FIG. 8. Occupation of the valence Kohn-Sham orbitals of benzene during interaction with a 10 -cycle linearly polarized Ti-sapphire $(\lambda=780 \mathrm{~nm})$ laser pulse having a peak intensity of $4.0 \times 10^{14} \mathrm{~W} / \mathrm{cm}^{2}$. The molecule lies in the $x-y$ plane and the laser-polarization lies along the $x$-axis (parallel to the molecular plane). For clarity only the HOMO (a) and HOMO (b) orbital populations are labeled, with the response of all other orbitals shown in red. We see that the response of the HOMO (b) orbital is suppressed with respect to the HOMO (a) orbital. 


\section{CONCLUSIONS}

In this paper we have presented a NAQMD approach for treating laser-molecule interactions. The approach is of sufficient generality that the response of a wide range of molecules can be studied: in particular both all-electron and pseudopotential calculations can be performed. Using this approach we were able to study the role of multielectron effects in HHG in $\mathrm{N}_{2}$ and benzene and show how the symmetry properties of the Kohn-Sham orbitals play an important role in the observed spectra. To the best of our knowledge, the results for benzene represent the first TDDFT calculations of HHG in benzene using linearly polarized laser pulses and suggest a suppression of the harmonic signal when the laser polarization direction is aligned perpendicular to the molecular plane.

The results presented here consider only fixed nuclei and exchange-correlation is only treated at the level of the LDA. However, our results are able to capture important aspects of the molecular response to the laser pulse. Subsequent studies will consider dynamical ion calculations. This will allow charge transfer across molecules during interaction with the laser pulse to be studied ${ }^{120,121}$ as well as how this transfer influences dissociation of the molecule. In addition, asymptotically corrected exchange-correlation potentials ${ }^{122}$ will be employed and this will be used to assess the accuracy of the excited states properties in our calculations and their importance in $\mathrm{HHG}^{44}$

Another important extension to the approach is the incorporation of transport boundary conditions that will allow current-flow through molecular electronic devices to be considered. Several schemes for achieving this are being studied. ${ }^{123-125}$

${ }^{1}$ A. H. Zewail, Femtochemistry: Ultrafast Dynamics of the Chemical Bond (World Scientific, Singapore, 1994).

${ }^{2}$ C. Joachim, J. K. Gimzewski, and A. Aviram, Nature (London) 408, 541 (2000).

${ }^{3}$ D. M. Willard and A. van Orden, Nature Mater. 2, 575 (2003).

${ }^{4}$ S. Kohler and P. Hänggi, Nat. Nanotechnol. 2, 675 (2007).

${ }^{5}$ I. Franco, M. Shapiro, and P. Brumer, Phys. Rev. Lett. 99, 126802 (2007).

${ }^{6}$ G.-Q. Li, M. Schreiber, and U. Kleinekathöfer, Europhys. Lett. 79, 27006 (2007).

${ }^{7}$ T. Brixner and G. Gerber, ChemPhysChem 4, 418 (2003).

${ }^{8}$ M. Wollenhaupt, T. Bayer, M. Krug, C. Sarpe-Tudoran, and T. Baumert, J. Phys.: Conf. Series 88, 012053 (2007).

${ }^{9}$ T. Laarmann, I. Shchatsinin, P. Singh, N. Zhavoronkov, C. P. Schulz, and I. V. Hertel, J. Phys. B 41, 074005 (2008).

${ }^{10}$ M. F. Kling, C. Siedschlag, A. J. Verhoef, J. I. Khan, M. Schultze, T. Uphues, Y. Ni, M. Uiberacker, M. Drescher, F. Krausz, and M. J. J. Vrakking, Science 312, 246 (2006).

${ }^{11}$ B. Fischer, M. Kremer, T. Pfeifer, B. Feuerstein, V. Sharma, U. Thumm, C. D. Schröter, R. Moshammer, and J. Ullrich, Phys. Rev. Lett. 105, 223001 (2010).

${ }^{12}$ M. Kremer, B. Fischer, B. Feuerstein, V. L. B. de Jesus, V. Sharma, C. Hofrichter, A. Rudenko, U. Thumm, C. D. Schröter, R. Moshammer, and J. Ullrich, Phys. Rev. Lett. 103, 213003 (2009).

${ }^{13}$ A. Baltuška, T. Udem, M. Uiberacker, M. Hentschel, E. Goulielmakis, C. Gohle, R. Holzwarth, V. S. Yakovlev, A. Scrinzi, T. W. Hänsch, and F. Krausz, Nature (London) 421, 611 (2003).

${ }^{14}$ M. Hentschel, R. Kienberger, C. Spielmann, G. A. Reider, N. Milosevic, T. Brabec, P. Corkum, U. Heinzmann, M. Drescher, and F. Krausz, Nature (London) 414, 509 (2001).

${ }^{15}$ P. M. Paul, E. S. Toma, P. Breger, G. Mullot, F. Augé, P. Balcou, H. G. Muller, and P. Agostini, Science 292, 1689 (2001).
${ }^{16}$ M. Drescher, M. Hentschel, R. Kienberger, G. Tempea, C. Spielmann, G. A. Reider, P. B. Corkum, and F. Krausz, Science 291, 1923 (2001).

${ }^{17}$ F. He, A. Becker, and U. Thumm, Phys. Rev. Lett. 101, 213002 (2008).

${ }^{18}$ G. Sansone, F. Kelkensberg, J. F. Pérez-Torres, F. Morales, M. F. Kling, W. Siu, O. Ghafur, P. Johnsson, M. Swoboda, E. Benedetti, F. Ferrari, F. Lépine, J. L. Sanz-Vicario, S. Zherebtsov, I. Znakovskaya, A. L'Huillier, M. Y. Ivanov, F. M. M. Nisoli, and M. J. J. Vrakking, Nature (London) 465, 763 (2010).

${ }^{19}$ K. P. Singh, F. He, P. Ranitovic, W. Cao, S. De, D. Ray, S. Chen, U. Thumm, A. Becker, M. M. Murnane, H. C. Kapteyn, I. V. Litvinyuk, and C. L. Cocke, Phys. Rev. Lett. 104, 023001 (2010).

${ }^{20}$ F. Krausz and M. Ivanov, Rev. Mod. Phys. 81, 163 (2009).

${ }^{21}$ A. Talebpour, C.-Y. Chien, and S. L. Chin, J. Phys. B 29, L677 (1996).

${ }^{22}$ C. Guo, M. Li, J. P. Nibarger, and G. N. Gibson, Phys. Rev. A 58, R4271 (1998).

${ }^{23}$ J. Muth-Böhm, A. Becker, and F. H. M. Faisal, Phys. Rev. Lett. 85, 2280 (2000).

${ }^{24}$ M. J. DeWitt, E. Wells, and R. R. Jones, Phys. Rev. Lett. 87, 153001 (2001).

${ }^{25}$ X. Chu and S.-I. Chu, Phys. Rev. A 70, 061402 (2004).

${ }^{26}$ D. Dundas and J. M. Rost, Phys. Rev. A 71, 013421 (2005).

${ }^{27}$ A. Jaroń-Becker, A. Becker, and F. H. M. Faisal, Phys. Rev. Lett. 96, 143006 (2006).

${ }^{28}$ Y. H. Jiang, T. Pfeifer, A. Rudenko, O. Herrwerth, L. Foucar, M. Kurka, K. U. Kühnel, M. Lezius, M. F. Kling, X. Liu, K. Ueda, S. Düsterer, R. Treusch, C. D. Schröter, R. Moshammer, and J. Ullrich, Phys. Rev. A 82, 041403 (2010).

${ }^{29}$ S.-I. Chu and M. McIntyre, Phys. Rev. A 83, 013409 (2011).

${ }^{30}$ N. Hay, R. de Nalda, T. Halfmann, K. J. Mendham, M. B. Mason, M. Castillejo, and J. P. Marangos, Phys. Rev. A 62, 041803 (2000).

${ }^{31}$ P. Žďánská, V. Averbukh, and N. Moiseyev, J. Chem. Phys. 118, 8726 (2003).

${ }^{32}$ R. Baer, D. Neuhauser, P. R. Žďánská, and N. Moiseyev, Phys. Rev. A 68, 043406 (2003).

${ }^{33}$ F. Ceccherini and D. Bauer, Phys. Rev. A 64, 033423 (2001).

${ }^{34}$ R. de Nalda, E. Heesel, M. Lein, N. Hay, R. Velotta, E. Springate, M. Castillejo, and J. P. Marangos, Phys. Rev. A 69, 031804 (2004).

${ }^{35}$ B. K. McFarland, J. P. Farrell, P. H. Bucksbaum, and M. Gühr, Science 322, 1232 (2008).

${ }^{36}$ N. Kajumba, R. Torres, J. G. Underwood, J. S. Robinson, S. Baker, J. W. G. Tisch, R. de Nalda, W. A. Bryan, R. Velotta, C. Altucci, I. Procino, and I. C. E. T. J. P. Marangos, New J. Phys. 10, 025008 (2008).

${ }^{37}$ A.-T. Le, R. R. Lucchese, and C. D. Lin, J. Phys. B 42, 211001 (2009).

${ }^{38}$ G. H. Lee, I. J. Kim, S. B. Park, T. K. Kim, Y. S. Lee, and C. H. Nam, J. Phys. B 43, 205602 (2010).

${ }^{39}$ D. A. Telnov and S.-I. Chu, Phys. Rev. A 80, 043412 (2009).

${ }^{40}$ G. Lagmago Kamta and A. D. Bandrauk, Phys. Rev. A 80, 041403 (2009).

${ }^{41}$ O. Smirnova, Y. M. S. Patchkovskii, N. Dudovich, D. Villeneuve, P. Corkum, and M. Y. Ivanov, Nature (London) 460, 972 (2009).

${ }^{42}$ O. Smirnova, S. Patchkovskii, Y. Mairesse, N. Dudovich, D. Villeneuve, P. Corkum, and M. Y. Ivanov, Phys. Rev. Lett. 102, 063601 (2009).

${ }^{43}$ O. Smirnova, S. Patchkovskii, Y. Mairesse, N. Dudovich, and M. Y. Ivanov, Proc. Natl. Acad. Sci. U.S.A. 106, 16556 (2009).

${ }^{44}$ Y. Mairesse, J. Higuet, N. Dudovich, D. Shafir, B. Fabre, E. Mével, E. Constant, S. Patchkovskii, Z. Walters, M. Y. Ivanov, and O. Smirnova, Phys. Rev. Lett. 104, 213601 (2010).

${ }^{45}$ C. Vozzi, R. Torres, M. Negro, L. Brugnera, T. Siegel, C. Altucci, R. Velotta, F. Frassetto, L. Poletto, P. Villoresi, S. D. Silvestri, S. Stagira, and J. P. Marangos, App. Phys. Lett. 97, 241103 (2010).

${ }^{46}$ J. Heslar, D. Telnov, and S.-I. Chu, Phys. Rev. A 83, 043414 (2011).

${ }^{47}$ E. P. Fowe and A. D. Bandrauk, Phys. Rev. A 81, 023411 (2010).

${ }^{48}$ E. P. Fowe and A. D. Bandrauk, Phys. Rev. A 84, 035402 (2011).

${ }^{49}$ J. Itatani, J. Levesque, D. Zeidler, H. Niikura, H. Pépin, J. C. Kieffer, P. B. Corkum, and D. M. Villeneuve, Nature (London) 432, 867 (2004).

${ }^{50}$ J. P. Marangos, S. Baker, N. Kajumba, J. S. Robinson, J. W. G. Tisch, and R. Torres, Phys. Chem. Chem. Phys. 10, 35 (2008).

${ }^{51}$ S. Chelkowski, T. Zuo, and A. D. Bandrauk, Phys. Rev. A 46, R5342 (1992).

${ }^{52}$ H. Kono, Y. O. A. Kita, and Y. Fujimura, J. Comput. Phys. 130, 148 (1997).

${ }^{53}$ M. Lein, P. P. Corso, J. P. Marangos, and P. L. Knight, Phys. Rev. A 67, 023819 (2003).

${ }^{54}$ D. Dundas, K. J. Meharg, J. F. McCann, and K. T. Taylor, Eur. Phys. J. D 26, 51 (2003) 
${ }^{55}$ S. Chelkowski, C. Foisy, and A. D. Bandrauk, Phys. Rev. A 57, 1176 (1998).

${ }^{56}$ A. D. Bandrauk and H. Lu, Int. J. Quantum Chem. 99, 452 (2004).

${ }^{57}$ Y. V. Vanne and A. Saenz, Phys. Rev. A 82, 011403(R) (2010).

${ }^{58}$ E. Runge and E. K. U. Gross, Phys. Rev. Lett. 52, 997 (1984).

${ }^{59}$ U. Saalmann and R. Schmidt, Z. Phys. D 38, 153 (1996).

${ }^{60}$ U. Saalmann and R. Schmidt, Phys. Rev. Lett. 80, 3213 (1998).

${ }^{61}$ T. Kunert and R. Schmidt, Eur. Phys. J. D 25, 15 (2003).

${ }^{62}$ M. Uhlmann, T. Kunert, F. Grossmann, and R. Schmidt, Phys. Rev. A 67, 013413 (2003)

${ }^{63}$ D. Dundas, J. Phys. B 37, 2883 (2004).

${ }^{64}$ F. Calvayrac, C. A. U. P.-G. Reinhard, and E. Suraud, Phys. Rep. 337, 493 (2000).

${ }^{65}$ C. A. Altucci, M. A. L. Marques, J. A. Alonso, G. F. Bertsch, and A. Rubio, Eur. Phys. J. D 28, 211 (2004).

${ }^{66}$ F. Gygi, Europhys. Lett. 19, 617 (1992).

${ }^{67}$ A. Devenyi, K. Cho, T. A. Arias, and J. D. Joannopoulos, Phys. Rev. B 49, 13373 (1994)

${ }^{68}$ D. R. Hamann, Phys. Rev. B 51, 9508 (1995).

${ }^{69}$ E. Fattal, R. Baer, and R. Kosloff, Phys. Rev. E 53, 1217 (1996).

${ }^{70}$ J. M. Pérez-Jordá, Phys. Rev. B 58, 1230 (1998).

${ }^{71}$ F. Gygi and G. Galli, Phys. Rev. B 52, R2229 (1995).

${ }^{72}$ J. M. Pérez-Jordá, Phys. Rev. A 52, 2778 (1995).

${ }^{73}$ N. A. Modine, G. Zumbach, and E. Kaxiras, Phys. Rev. B 55, 10289 (1997).

${ }^{74}$ A. Castro, H. Appel, M. Oliveira, C. A. Rozzi, X. Andrade, F. Lorenzen, M. A. L. Marques, E. K. U. Gross, and A. Rubio, Phys. Status Solidi B 243, 2465 (2006).

${ }^{75}$ D. Dundas, J. F. McCann, J. S. Parker, and K. T. Taylor, J. Phys. B 33, 3261 (2000).

${ }^{76}$ T. N. Todorov, J. Phys.: Condens. Matter 13, 10125 (2001).

${ }^{77}$ T. A. Niehaus, D. Heringer, B. Torralva, and T. Frauenheim, Eur. Phys. J. D 35, 467 (2005).

${ }^{78}$ P. Pulay, Mol. Phys. 17, 153 (1969).

${ }^{79}$ M. Lein, T. Kreibich, E. K. U. Gross, and V. Engel, Phys Rev A 65, 033403 (2002).

${ }^{80}$ T. Kreibach, R. van Leeuwen, and E. K. U. Gross, Chem. Phys. 304, 183 (2004).

${ }^{81}$ W. Vanroose, F. Martín, T. N. Rescigno, and M. C. W, Science 310, 1787 (2005).

82 A. D. Bandrauk, S. Chelkowski, S. Kawai, and H. Lu, Phys. Rev. Lett. 101, 153901 (2008).

${ }^{83}$ I. Kawata and H. Kono, J. Phys. Chem. 111, 9498 (1999).

${ }^{84}$ F. Gygi, Phys. Rev. B 48, 11692 (1993).

${ }^{85}$ J. R. Chelikowsky, N. Troullier, K. Wu, and Y. Saad, Phys. Rev. B 50, 11355 (1994).

${ }^{86}$ T. L. Beck, Rev. Mod. Phys. 72, 1041 (2000).

${ }^{87}$ D. R. Hamann, Phys. Rev. B 63, 075107 (2001).

${ }^{88}$ W. E. Arnoldi, Q. Appl. Math. 9, 17 (1951).

${ }^{89}$ E. S. Smyth, J. S. Parker, and K. T. Taylor, Comput. Phys. Commun. 114, 1 (1998).

${ }^{90}$ D. C. Liu and J. Nocedal, Math. Program. 45, 503 (1989).

${ }^{91}$ D. G. Anderson, J. Assoc. Comput. Mach. 12, 547 (1964).

${ }^{92}$ P. Pulay, Chem. Phys. Lett. 73, 393 (1980).

${ }^{93}$ E. R. Hernández, S. Janecek, M. Kaczmarski, and E. Krotscheck, Phys. Rev. B 75, 075108 (2007).

${ }^{94}$ C. Lanczos, J. Res. Natl. Bur. Stand. 45, 255 (1950).
${ }^{95}$ C. Bekas, J. R. C. Y. Saad, and M. L. Tiago, Comput. Phys. Commun. 171, 175 (2005).

${ }^{96}$ K. Wu, A. Canning, H. D. Simon, and L.-W. Wang, J. Comput. Phys. 154, 156 (1999).

${ }^{97}$ Y. Zhou, Y. Saad, M. L. Tiago, and J. R. Chelikowsky, J. Comput. Phys. 219, 172 (2006)

${ }^{98}$ Y. Zhou, Y. Saad, M. L. Tiago, and J. R. Chelikowsky, Phys. Rev. E 74, 066704 (2006).

${ }^{99}$ J. Kohanoff, and N. Gidopoulos, in Handbook of Molecular Physics and Quantum Chemistry, edited by S. Wilson (Wiley, 2003), Vol. 2, p. 532.

${ }^{100} \mathrm{~N}$. Troullier and J. L. Martins, Phys. Rev. B 43, 1993 (1991).

${ }^{101}$ L. Kleinman and D. M. Bylander, Phys. Rev. Lett. 48, 1425 (1982).

${ }^{102}$ X. Jing, N. Troullier, D. Dean, N. Binggeli, J. R. Chelikowsky, K. Wu, and Y. Saad, Phys. Rev. B 50, 12234 (1994).

${ }^{103}$ M. Oliveira and F. Nogueira, Comput. Phys. Commun. 178, 524 (2008).

${ }^{104}$ T. Grabo, T. Kreibich, S. Kurth, and E. Gross, in Strong Coulomb Correlations in Electronic Structure Calculations: Beyond the Local Density Approximation, edited by V. I. Anisimov (Gordon and Breach, 2000), p. 203.

${ }^{105}$ E. J. Meijer and M. Sprik, J. Chem. Phys. 105, 8684 (1996).

${ }^{106}$ S. Parthiban and J. M. L. Martin, J. Chem. Phys. 115, 2051 (2001).

${ }^{107}$ S. M. Sharifi, A. Talebpour, and S. L. Chin, J. Phys. B 40, F259 (2007).

${ }^{108}$ L. Lehtovaara, V. Havu, and M. Puska, J. Chem. Phys. 131, 054103 (2009).

${ }^{109}$ S.-K. Son, J. Comput Phys. 230, 2160 (2011).

${ }^{110}$ P. B. Corkum, Phys. Rev. Lett. 71, 1994 (1993).

${ }^{111}$ J. L. Krause, K. J. Schafer, and K. C. Kulander, Phys. Rev. Lett. 68, 3535 (1992).

${ }^{112}$ K. Burnett, V. C. Reed, J. Cooper, and P. L. Knight, Phys. Rev. A 45, 3347 (1992).

${ }^{113}$ M. Lewenstein, P. Balcou, M. Y. Ivanov, A. L'Huillier, and P. B. Corkum, Phys. Rev. A 49, 2117 (1994).

${ }^{114}$ S. Petretti, Y. V. Vanne, A. Saenz, A. Castro, and P. Decleva, Phys. Rev. Lett. 104, 223001 (2010).

${ }^{115}$ C. Jin, J. B. Bertrand, R. R. Lucchese, H. J. Wörner, P. B. Corkum, D. M. Villeneive, A.-T. Le, and C. D. Lin, Phys. Rev. A 85, 013405 (2012).

${ }^{116}$ Y. Mairesse, S. Haessler, B. Fabre, J. Higuet, W. Boutu, P. Breger, E. Constant, D. Descamps, E. Mével, S. Petit, and P. Salières, New J. Phys. 10, 025208 (2008).

${ }^{117}$ S. Haessler, J. Caillat, W. Boutu, C. Giovanetti-Teixeira, T. Ruchon, T. Auguste, Z. Diveki, P. Breger, A. Maquet, B. Carré, and R. T. P. Salières, Nat. Phys. 6, 200 (2010).

${ }^{118}$ A. Rupenyan, J. B. Bertrand, D. M. Villeneuve, and H. J. Wörner, Phys. Rev. Lett. 108, 033903 (2012).

${ }^{119}$ B. A. Sickmiller and R. R. Jones, Phys. Rev. A 80, 031802 (2009).

${ }^{120}$ F. Remacle and R. D. Levine, Proc. Natl. Acad. Sci. U.S.A. 103, 6793 (2006).

${ }^{121}$ S. Lünnermann, A. I. Kuleff, and L. S. Cederbaum, J. Chem. Phys. 129, 104305 (2008).

${ }^{122}$ R. van Leeuwen and E. J. Baerends, Phys. Rev. A 49, 2421 (1994).

${ }^{123}$ E. J. McEniry, D. R. Bowler, D. Dundas, A. P. Horsfield, C. G. Sánchez, and T. N. Todorov, J. Phys.: Condens. Matter 19, 196201 (2007).

${ }^{124}$ G. Stefanucci, S. Kurth, A. Rubio, and E. K. U. Gross, Phys. Rev. B 77, 075339 (2008).

${ }^{125}$ C.-L. Cheng, J. S. Evans, and T. V. Voorhis, Phys. Rev. B 74, 155112 (2006). 